\title{
Massless Phases and Symmetry Restoration in Abelian Gauge Theories and Spin Systems
}

\author{
Jürg Fröhlich ${ }^{1 \star}$ and Thomas Spencer ${ }^{2 \star \star}$ \\ 1 Institut des Hautes Etudes Scientifiques, 35, Route de Chartres, F-91440 Bures-sur-Yvette, France \\ 2 Courant Institute of Mathematical Sciences, New York University, 251 Mercer Street, New York, \\ NY 10012, USA
}

\begin{abstract}
We give a new, elementary proof for the existence of a deconfining transition to a massless (QED) phase in the four-dimensional $U(1)$ lattice gauge theory and of an intermediate QED phase, accompanied by dynamical restoration of local $U(1)$ invariance, in the four dimensional $\mathbb{Z}_{N}$ models, with $N$ large. Our methods can also be used to prove the existence of a phase transition in the $X Y$ model in three or more dimensions, in three- and four-dimensional abelian Higgs models, and in more general models admitting some local, abelian gauge invariance.
\end{abstract}

\section{Introduction and Summary of Results}

In the past five years, there has been considerable progress in the understanding of the phase diagram of lattice gauge theories with a discrete (abelian, or non-abelian) "unbroken" group of gauge transformations. Among such models are

i) pure lattice gauge theories with a discrete gauge group;

ii) lattice Higgs models with discrete or continuous gauge groups, broken down by the Higgs scalars to a discrete, unbroken subgroup.

Such models are now known to have a strong coupling ("high temperature") phase in which static quarks transforming non-trivially under the center of the unbroken group are confined and a weak coupling (or "low temperature") phase where static quarks are not confined but magnetic monopoles may be; see [1-6] for a systematic review and further developments.

Proofs of these results are based on fairly standard high and low temperature expansions. An excellent review of such expansions $[7,8]$ along with applications to lattice gauge theories can be found in [6]. None of these expansion methods require the use of duality transformations, so that non-abelian models with discrete, unbroken groups are accessible. The applications to the study of Higgs models with continuous gauge groups, but discrete unbroken subgroups is

* Address after Aug. 1982: Physics Dept., ETH, Zürich, Switzerland

$\star \star$ Work supported in part by the NSF under grant DMR 81-00417 
somewhat subtle. However, the methods developed in $[9,10]$, adapted to lattice gauge theories, are in principle sufficient to study such models in various, extreme regions of coupling constant space; see also [6].

As an example, consider a four-dimensional SU(2) Higgs model with a system of Higgs scalars which leave only $\mathbb{Z}_{2}$ unbroken. Let $g$ be the pure gauge coupling constant, $\beta \equiv 1 / g^{2}$, and suppose that, in the unitary gauge, the interaction between the lattice gauge field, $g$, and the matter fields is given by the action

$$
-\zeta R_{x} R_{y} \sum_{x y} \chi_{1}\left(g_{x y}\right)
$$

where $x y$ runs through all bonds (nearest neighbor pairs) of $\mathbb{Z}^{4}, \chi_{1}$ is the spin 1 character of $\mathrm{SU}(2), \zeta>0$ is a coupling constant, and $R_{x}$ is the radial component of the Higgs system at the point $x \in \mathbb{Z}^{4}$ which is supposed to be $\approx R_{0}>0$ with high probability.

Presently, those facts which are known rigorously about this model can be summarized in the following diagram:

I: Confinement of static quarks in the fundamental representation $[1,4]$.

II : Confinement of $\mathbb{Z}_{2}$ monopoles [6].

Fig. 1

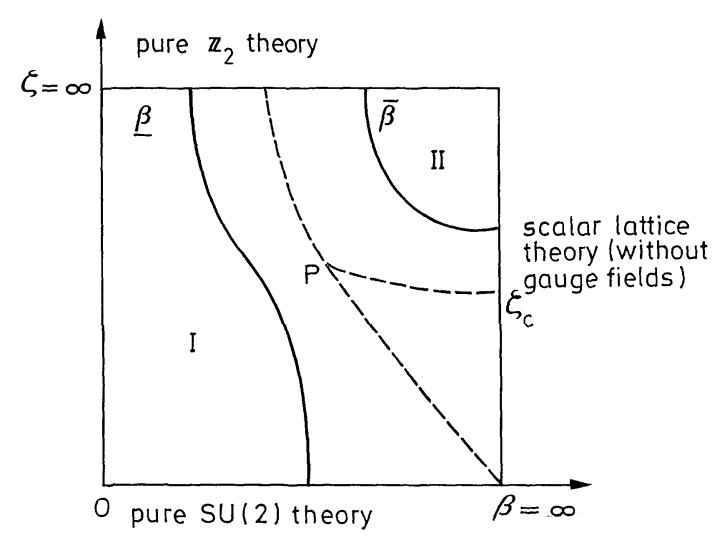

On the line $\beta=\infty$, the theory reduces to a lattice theory of scalar fields decoupled from the gauge fields which has in general a phase transition, with a massless, broken symmetry phase for $\zeta>\zeta_{c}[11]$.

It is conjectured that $\beta=\bar{\beta}$ and, more generally that regions I and II have a common boundary from $(\underline{\beta}=\bar{\beta}, \zeta=\infty)$ to some point $P$ which is connected by a line of singularities of e.g. the magnetic string tension to $\left(\beta=\infty, \zeta=\zeta_{c}\right)$. Moreover, domain I should extend to the broken line from $P$ to $(\beta=\infty, \zeta=0)$.

Among the obstructions which prevent one from proving the above conjectures are: incomplete knowledge of the pure $\mathbb{Z}_{2}$ theory; the presumed roughening transition in the pure $S U(2)$ theory (see e.g. [12]) which appears to make it impossible to extrapolate the high temperature expansion for $\zeta=0$ to arbitrarily large values of $\beta$. 
The model discussed above may be amusing, but is not really relevant for particle physics. More interesting examples would be lattice versions of the Georgi-Glashow or the standard (Glashow-) Weinberg-Salam model of electroweak interactions. In these models a new difficulty appears: essentially no powerful, analytical tools are known which would permit one to establish the existence of electromagnetic phases with massless photons and unconfined, charged leptons.

Let us consider, for example, the Georgi-Glashow model. In this model, the Higgs scalar has isotopic spin 1, and the action describing the interactions between the Higgs and the gauge field is given by

$$
-\zeta \sum_{x y}\left(\phi_{x}, D_{1}\left(g_{x y}\right) \phi_{y}\right)
$$

where $\phi$ is the Higgs field, $D_{1}$ is the spin 1 representation of $\operatorname{SU}(2),(\cdot, \cdot)$ is the scalar product on $\mathbb{R}^{3}, \zeta>0$.

In this example the presumed phase diagram is described in Fig. 2 below.

Fig. 2

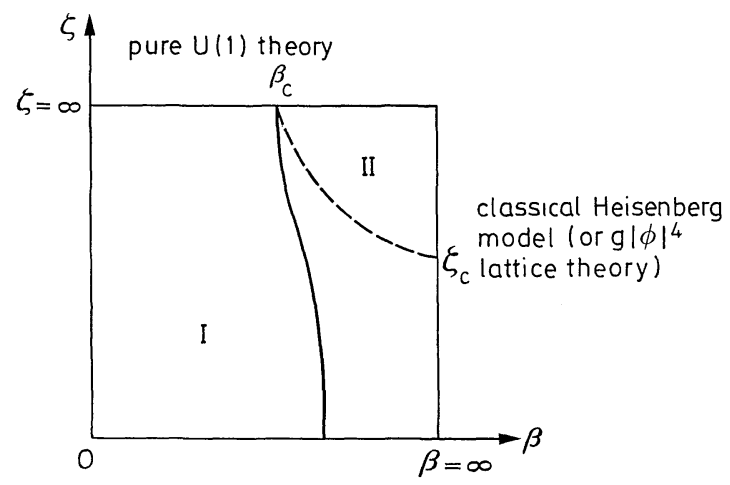

In domain I static "leptons" in the fundamental representation of SU(2) are confined. This follows from the results of $[1,6]$ (high temperature expansions) or from [4] (where correlation inequalities are used).

When $\zeta=\infty$ the model reduces to the pure $U(1)$ lattice theory. One main result of our paper is a new proof and a generalization of a result, already established by Guth [13], which asserts that the four-dimensional $U(1)$ model has a deconfining transition, i.e. for $\beta>\beta_{c}$, static electric charges have only Coulombic interactions, and the photon is massless (see Sect. 2).

Our method of proof is a descendant of a more involved one used to establish the existence of the Berezinski-Kosterlitz-Thouless transition [14] in the twodimensional rotator model and the Coulomb gas which we presented in [15]. In comparison with [15] simplifications arise in the analysis of the $U(1)$ model, due to gauge invariance which enforces "local neutrality". Our methods have the advantage over [13] of not being geared to a special form of the lattice action (the Villain action), and they do not involve a cluster expansion (so that reasonable bounds on $\beta_{c}$ might be obtained). Physically speaking, they consist in showing that for large $\beta$, static electric charges are deconfined, because the dynamical magnetic 
monopoles of the lattice $U(1)$-model are bound in neutral clusters which form a dilute gas.

On the line $\beta=\infty$, the model reduces to the classical Heisenberg model or the three-component lattice $g|\phi|^{4}$ theory, and the degrees of freedom of the gauge field are frozen. These models have a phase transition accompanied by spontaneous breaking of $O(3)$ : For $\zeta>\zeta_{c}$, global $O(3)$ invariance is broken, and there exist two massless Goldstone modes. This has been proven in [11]. (For two-component rotator models, a new proof of this result is given in Sect. 4.)

We expect that the critical points $\beta_{c}$ and $\zeta_{c}$ are connected by a line of critical points above which the theory is in a massless QED phase with unconfined electric charge and massive, magnetic monopoles (see domain II, Fig. 2). In the complement of domain II, and for $\beta<\infty$, magnetic monopoles are expected to be massless. For $\zeta$ sufficiently small and $\beta$ below $\beta_{\text {roughening [for the pure } \mathrm{SU}(2) \text { theory] }}$ they are expected to form a condensate. In this range of parameters electric charge is confined. Since our analysis of the $U(1)$ model involves using a duality transformation, it does not extend to the model with $\zeta<\infty$, in any obvious way ${ }^{1}$. This and the absence of a detailed understanding of the presumed roughening transition in the pure $\mathrm{SU}(2)$ theory are, at present, the obstructions against establishing the conjectured phase diagram described above. At least, it is supported by the results in Sect. 2 and [11].

In Sect. 3, we reconsider the $\mathbb{Z}_{N}$ models with Villain or Wilson action. We show that, in four dimensions and for $N$ large enough, there exist two critical values of $\beta$, $\beta_{c}$, and $\bar{\beta}_{c}>\beta_{c}$ (depending on $\left.N\right)$, such that for $\beta \in\left(\beta_{c}, \bar{\beta}_{c}\right)$ the Wilson and the disorder loop have perimeter decay. Thus there exist intermediate QED phases. This reproduces and extends a result of Elitzur et al. [16]. The point of our methods is to avoid using self-duality which only holds for the Villain action and to exhibit a sequence of transformations of the $\mathbb{Z}_{N}$ model which map it to a model with unbroken $U(1)$ gauge invariance, provided $\beta \in\left(\beta_{c}, \bar{\beta}_{c}\right)$. In other words, local $U(1)$ invariance is restored in the intermediate phase. This is the analogue of global $U(1)$ restoration in the intermediate phases of the two-dimensional $\mathbb{Z}_{N}$ models which we described in [15].

The phenomenon that the "fixed point theory" of some class of spin systems or lattice gauge theories, with respect to suitably chosen renormalization transformations, has a larger global or local symmetry than the original models is presumably a rather general one. It is therefore of interest to analyze some examples which exhibit that phenomenon.

We expect it to occur, for example, in any lattice gauge theory with a discrete gauge group $H$ of high order which is a subgroup of some Lie group: If $G$ is the smallest Lie group containing $H$ as a subgroup, then we expect that a pure lattice gauge theory with gauge group $H$ has intermediate phases where local $G$-invariance is restored, in the sense that certain correlations behave like ones in a pure gauge theory with gauge group $G$.

In Sect. 4 we reconsider the classical rotator $(X Y)$ model in three or more dimensions. By duality, the rotator model is equivalent to a statistical mechanical

1 It is an interesting problem to avoid the use of duality in the analysis of the $U(1)$ model, or to translate the methods developed in Sect. 2 back into the Wilson formulation of that model 
model of line defects characterized by integer flux numbers. In three dimensions, this model is the $\zeta \rightarrow \infty$ limit of the non-compact, abelian Higgs model, and the line defects correspond to the Abrikosov vortices.

Our methods permit us to prove that, for a large class of lattice actions, the classical $X Y$ model in three- or more dimensions has a phase transition with long range order, accompanied by spontaneous symmetry breaking. By the results of [5] this also implies the existence of a superconductor $\rightarrow$ QED transition in the three-dimensional, abelian Higgs model. (In the superconducting phase, vortices have a small activity and form a dilute gas, the photon is massive and there is no confinement of fractionally charged static sources. In the QED phase, vortices condense, the photon is massless, and fractionally charged sources are confined by a logarithmic potential. These results were proven in [5], assuming the results of [15] and of Sect. 4 of the present paper, by using correlation inequalities.)

We conclude this introduction by establishing some notation: Let $G$ be a compact gauge group. With each link (nearest neighbor pair) $x y$ in a simple, cubic lattice $\mathbb{Z}^{D}$ we associate an element $g_{x y}$ of $G$. The a priori distribution of $g_{x y}$ is given by the normalized Haar measure, $d g_{x y}$, on $G$. Let $\Lambda$ be some finite region in $\mathbb{Z}^{D}$, and let $\chi$ be some unitary or orthogonal character of $G$, typically the character of the fundamental representation of $G$ (assumed here to be a matrix group).

Following Wilson [1], the action of a lattice theory in region $\Lambda$ is defined by

$$
A_{\beta}\left(g_{\Lambda}\right)=-\beta \sum_{p \subset A} \operatorname{Re} \chi\left(g_{\partial p}\right),
$$

where $\beta=1 / g^{2}$ is the inverse square of the gauge coupling constant, $p$ denotes a unit lattice square (plaquette) in $\Lambda, g_{\Lambda}$ is shorthand for $\left\{g_{x y}\right\}_{x y \subset A}$, and

$$
g_{\partial p}=\prod_{x y \subset \partial p} g_{x y} \text {. }
$$

Here $\prod$ denotes a path-ordered product. The Euclidean functional measure of the lattice theory in $\Lambda$ is given by

$$
d \mu_{\beta}\left(g_{\Lambda}\right)=Z_{\beta, \Lambda}^{-1} e^{-A_{\beta}\left(g_{\Lambda}\right)} \prod_{x y \subset \Lambda} d g_{x y} .
$$

More generally, $d \mu_{\beta}$ is defined by

$$
d \mu_{\beta}\left(g_{\Lambda}\right)=Z_{\beta, \Lambda}^{-1} \prod_{p \subset A} \varphi_{\beta}\left(g_{\partial p}\right) \prod_{x y \subset A} d g_{x y},
$$

where $\varphi_{\beta}$ is some positive class function on $G$, i.e.

$$
\varphi_{\beta}\left(h^{-1} g h\right)=\varphi_{\beta}(g) \text {. }
$$

For example, $\varphi_{\beta}$ may be the heat kernel on $G$ in which case the model is called the Villain approximation. In this paper, we primarily study the Villain approximation to the $U(1)$ model and the $X Y$ model, except in Sect. 3, where we study Wilson's form of the $\mathbb{Z}_{N}$ lattice gauge theory in four dimensions. This restriction is not inherent in our methods but is imposed for technical (mainly notational) convenience. The techniques introduced in Sect. 6 and Appendix B of [15] permit 
us to extend all results of the present paper to the models with Wilson action. (This is an advantage of our methods over the ones in [13].)

Our criterion for confinement (or deconfinement) of static sources is the usual Wilson criterion. We are aware of the shortcomings of this criterion. Instead, we could use the slightly more general criterion discussed in [5] which is correct in the limit of infinitely heavy "quarks". This would merely result in a slight complication of notations but does not alter our results. (It is an interesting open problem, not studied in this paper, to introduce a confinement criterion which is valid in theories with dynamical quarks of small mass.)

Let $\mathscr{L} \equiv \mathscr{L}_{L \times T}$ be a rectangular loop in a lattice plane, with sides of length $L$ and $T$. Let

$$
W(\mathscr{L})=\chi_{0}\left(\prod_{x y \subset \mathscr{L}} g_{x y}\right),
$$

where $\chi_{0}$ is some character of $G$.

Consider the expectation

$$
\langle W(\mathscr{L})\rangle_{\Lambda}(\beta) \equiv \int W(\mathscr{L}) d \mu_{\beta}\left(g_{\Lambda}\right), \quad \mathscr{L} \subset \Lambda .
$$

Let $\langle-\rangle(\beta) \equiv \lim _{\Lambda\urcorner_{\mathbb{Z}^{D}}}\langle-\rangle_{\Lambda}(\beta)$ denote the vacuum functional in the thermodynamic limit. (Some limit always exists by compactness ${ }^{2}$.) The "quark-anti-quark" potential is defined by

$$
V_{0}(L)=\lim _{T \rightarrow \infty}-\frac{1}{T} \log \left\langle W\left(\mathscr{L}_{L \times T}\right)\right\rangle(\beta) .
$$

(For a more accurate definition see [5].) Quarks transforming under a representation of $G$ with character $\chi_{0}$ are expected to be permanently confined if

$$
V_{0}(L) \text { diverges to }+\infty \text {, as } L \rightarrow \infty \text {. }
$$

This is possible only if $\chi_{0}$ is non-trivial on the center of $G$ [18]. Moreover,

$$
V_{0}(L) \leqq \text { const } L
$$

for arbitrary $G, \chi, \chi_{0}[19]$.

If

$$
\lim _{L \rightarrow \infty} V_{0}(L)<\infty
$$

"quarks" are expected to be deconfined, and physical states transforming nontrivially under the action of global gauge transformations corresponding to certain elements in the center of $G$ are expected to exist. While this conclusion is correct in a pure lattice gauge theory without dynamical quarks, it is wrong in theories with dynamical quarks in which (1.12) is valid in general, although quarks may be permanently confined. In order to establish the existence of a QED phase in the four-dimensional $U(1)$ model, one should therefore really also establish the masslessness of the photon (see Sect. 2).

2 In the abelian case, the existence of the limit follows from [17] 
For (1.12) to hold it suffices that

$$
\left\langle W\left(\mathscr{L}_{L \times T}\right)\right\rangle_{\Lambda}(\beta) \geqq \exp [-d(L+T)],
$$

for some $\Lambda$-independent constant $d$, provided $\Lambda$ is large enough. Inequality (1.13) is proven in the next section for the $U(1)$ model in four dimensions, at large values of $\beta$.

Apart from the behaviour of the Wilson loop expectation, $\langle W(\mathscr{L})\rangle(\beta)$, we are also interested in the behaviour of the expectation value of the disorder loop, $D$, in the state $\langle-\rangle(\beta)$. In four dimensions $D$ is defined as follows: One chooses a loop, $\mathscr{L}$, in a coordinate plane of the lattice $\left(\mathbb{Z}^{4}\right)^{*}$, dual to $\mathbb{Z}^{4}$. Let $\Sigma$ be an arbitrary set of plaquettes bounded by $\mathscr{L}$, i.e. $\partial \Sigma=\mathscr{L}$, and let

$$
\Sigma^{*}=\left\{p \subset \mathbb{Z}^{4}: p^{*} \subset \Sigma\right\} .
$$

Then

$$
\left\langle D_{\mathscr{L}}\right\rangle=\int \prod_{p \subset \Sigma^{*}}\left(\varphi_{\beta}\left(g_{\partial p} z\right) / \varphi_{\beta}\left(g_{\partial p}\right)\right) d \mu_{\beta}\left(g_{\Lambda}\right),
$$

where $z$ is an arbitrary, non-trivial element in the center of $G$. It has been shown in [5] that in the four-dimensional Villain approximation to the $U(1)$ model

$$
\left\langle D_{\mathscr{L}_{L \times T}}\right\rangle(\beta) \geqq \exp [-\delta(L+T)],
$$

for all $\beta<\infty$. This can also be shown for the $U(1)$ model with Wilson action by using the method of real translations (Sects. 5-7 of [15]) and Jensen's inequality. Thus, in the $U(1)$ model, the disorder loop always has perimeter decay, i.e. static magnetic monopoles are never confined.

In Sect. 3 we show that for sufficiently large $N$, the $\mathbb{Z}_{N}$ models with Wilson action have an intermediate phase [for $\beta \in\left(\underline{\beta}_{c}, \bar{\beta}_{c}\right)$, with $0<\beta_{c}<\bar{\beta}_{c}<\infty$ ] in which both inequalities, (1.13) and (1.15), hold. [It follows from standard high temperature expansions that (1.13) fails for small $\beta$ and (1.15) for large $\beta$, for every $N<\infty$.]

In Sect. 4 we extend the concepts and results described above to a general class of abelian models, "hypergauge theories", which includes the rotator model. We determine the (lower) critical dimension of these models.

\section{The Transition in the Four-Dimensional $U(1)$-Model}

\subsection{Main Ideas}

In this section we establish the existence of a transition to a deconfining, massless phase in the four-dimensional, compact $U(1)$ lattice gauge theory. Previous work concerning this model is contained in $[1,20,5]$ and, in particular in [13]. (See also [6] for a review of [13].)

The basic ideas of our method which evolved from [20] and [15] are as follows:

i) Use of Fourier transformation in the angular variables of the compact $U(1)$ model: Transformation to the non-compact, dual model.

ii) Application of a sequence of renormalization transformations to the dual model which map it to a neighborhood of the Gaussian model which describes 
free, non-compact electromagnetism. Our transformations represent a simplified version of the ones used in the two-dimensional Coulomb gas, in order to establish the existence of the Kosterlitz-Thouless transition [15]. The simplifications arise as a consequence of gauge invariance.

iii) Change of field variables in the renormalized dual models (real translations; see Sects. 5-7 of [15]) and application of Jensen's inequality to establish a lower bound on the Wilson loop expectation, i.e. the disorder loop expectation of the dual model, with perimeter decay. [This proves (1.13).]

\subsection{Notation and Definitions}

We explain our methods in terms of the Villain approximation to the $U(1)$ model, but with some analytical complications taken into account (see Sect. 6 and Appendix B of [15]) our methods and results extend to a large class of $U(1)$ models with other actions, in particular the Wilson action, as well.

In this and the following sections we use the notation

$$
g_{x y}=e^{i \theta_{x y}}, \quad \theta_{x y} \in[-\pi, \pi),
$$

to denote the elements of (subgroups of) $U(1)$. We adopt the usual convention

$$
\theta_{y x}=-\theta_{x y} .
$$

The a priori distribution of $\theta_{x y}$ is given by the Lebesgue measure, $d \theta_{x y}$, on the unit circle. Let $\Lambda$ be a finite, rectangular array of sites in $\mathbb{Z}^{4}$, and $\theta_{\Lambda}=\left\{\theta_{x y}\right\}_{x y c \Lambda}$, as in Sect. 1 . We define

$$
\varphi_{\beta}(\theta)=\sum_{n \in \mathbb{Z}} \exp \left[-(\beta / 2)(\theta+2 \pi n)^{2}\right], \quad \theta \in[-\pi, \pi) .
$$

This is the heat kernel on the unit circle appearing in the definition of the Villain approximation.

The purpose of this section is to elucidate the properties of the following distribution (the Euclidean functional measure for compact QED on the lattice):

$$
d \mu_{\beta}\left(\theta_{\Lambda}\right)=\hat{Z}_{\Lambda}^{-1} \prod_{p \subset \Lambda} \varphi_{\beta}\left(d \theta_{p}\right) \prod_{x y \subset \Lambda} d \theta_{x y}
$$

where

$$
d \theta_{p}=\sum_{x y \subset \partial p} \theta_{x y}
$$

( $\partial p$ is the boundary of a plaquette $p \subset \Lambda$ ), and

$$
\hat{Z}_{\Lambda}=\int \prod_{p \subset \Lambda} \varphi_{\beta}\left(d \theta_{p}\right) \prod_{x y \subset \Lambda} d \theta_{x y} .
$$

The standard Wilson loop is defined by

$$
W(\mathscr{L})=\prod_{x y \mathcal{L}} e^{i \theta_{x y}},
$$


where $\mathscr{L}$ is as in Sect. 1 , and we assume it to lie in the $0-1$ lattice plane. More generally, let

$$
W_{m}(\mathscr{L})=\prod_{x y \subset L} e^{i m \theta_{x y}}, \quad W_{m=1} \equiv W
$$

We now define

and

$$
\left.\begin{array}{rl}
\left\langle W_{m}(\mathscr{L})\right\rangle_{\Lambda}(\beta) & =\int W_{m}(\mathscr{L}) d \mu_{\beta}\left(\theta_{\Lambda}\right), \\
\left\langle W_{m}(\mathscr{L})\right\rangle(\beta) & =\lim _{\Lambda>\mathbb{Z}^{4}}\left\langle W_{m}(\mathscr{L})\right\rangle_{\Lambda}(\beta) .
\end{array}\right\}
$$

Existence of the limit is a standard consequence of Ginibre's inequalities [17] (for the models with Wilson's and with Villain action [21]). By a standard high temperature expansion (see e.g. [1]) or by using Simon's correlation inequalities $[22,23]$ one shows that, for $\beta$ sufficiently small,

with

$$
\left.\begin{array}{rl}
0 & \leqq\left\langle W_{m}(\mathscr{L})\right\rangle(\beta) \leqq \exp [-c(m, \beta) L \cdot T], \\
\beta) & \approx \ln \beta^{-1}, \text { as } \beta \rightarrow 0 .
\end{array}\right\}
$$

In the following, we propose to give a simple proof of the statement that, for $\beta$ large enough,

$$
\left\langle W_{m}(\mathscr{L})\right\rangle(\beta) \geqq \exp [-d(m, \beta)(L+T)],
$$

for some finite constant $d(m, \beta)$.

For reasons of simplicity of the exposition we concentrate on the model with Villain action and $m=1$, but using some results in [15] it is not challenging to extend our arguments to the general case. We now pause to review some exterior difference calculus.

\subsection{Exterior Difference Calculus}

Let $c_{k}$ denote an oriented unit $k$-cell in a simple, hypercubic lattice $\mathbb{Z}^{D}$. Let $\alpha$ be a $k$-form, i.e.

$$
\alpha: c_{k} \rightarrow \alpha\left(c_{k}\right) \in K,
$$

where $K$ is a ring, $(K=\mathbb{Z}, \mathbb{R}$ or $\mathbb{C})$, and $\alpha\left(c_{k}\right)=0$, except for finitely many $c_{k}$. We let $c_{k}^{-}$denote the same $k$-cell as $c_{k}$, but with orientation reversed, and require that

$$
\alpha\left(c_{k}^{-}\right)=-\alpha\left(c_{k}\right)
$$

Given an oriented $(k+1)$-cell, $c_{k+1}$, we define

$$
(d \alpha)\left(c_{k+1}\right)=\sum_{c_{k} \subset \partial c_{k+1}} \alpha\left(c_{k}\right)
$$

Here it is assumed that the orientation of some $c_{k} C \partial c_{k+1}$ is the one prescribed by the orientation of $c_{k+1}$, and (2.10) is enforced. Let $c_{k-1}$ be an oriented $(k-1)$-cell. We set

$$
(\delta \alpha)\left(c_{k-1}\right)=\sum_{c_{k}: \partial c_{k} \supset c_{k-1}} \alpha\left(c_{k}\right)
$$


assuming again that the orientations of the $\partial c_{k}$ 's are matched to the one of $c_{k-1}$ and (2.10) is enforced. Clearly, $d \alpha$ is a $(k+1)$-form, while $\delta \alpha$ is a $(k-1)$-form. One verifies easily that

$$
d d \alpha=0
$$

For,

$$
d(d \alpha)\left(c_{k+2}\right)=\sum_{c_{k+1} \subset \partial c_{k+2}}\left(\sum_{c_{k} \subset \partial c_{k+1}} \alpha\left(c_{k}\right)\right)
$$

Now, with each $c_{k}$ appearing in some $\partial c_{k+2}, c_{k}^{-}$appears in the same $\partial c_{k+2}$, too. Thus, by (2.10), the right side vanishes. Given arbitrary $k$-forms $\alpha$ and $\beta$, we set

$$
(\alpha, \beta)=\sum_{c_{k}}^{\prime} \overline{\alpha\left(c_{k}\right)} \beta\left(c_{k}\right),
$$

where $\alpha$ and $\beta$ are arbitrary $k$-forms, and $\sum^{\prime}$ extends over all positively oriented $k$-cells. One has

$$
(\beta, d \alpha)=(\delta \beta, \alpha)
$$

where $\alpha$ is an arbitrary $k$-form and $\beta$ an arbitrary $(k+1)$-form. This identity is a consequence of "summation by parts":

$$
\begin{aligned}
(\beta, d \alpha) & =\sum_{c_{k+1}} \overline{\beta\left(c_{k+1}\right)}(d \alpha)\left(c_{k+1}\right) \\
& =\sum_{c_{k+1}}^{\prime}\left(\sum_{c_{k} \subset \partial c_{k+1}} \overline{\beta\left(c_{k+1}\right)} \alpha\left(c_{k}\right)\right) \\
& =\sum_{c_{k}}\left(\sum_{c_{k+1}: \partial c_{k+1} \supset c_{k}} \overline{\beta\left(c_{k+1}\right)}\right) \alpha\left(c_{k}\right) \\
& =(\delta \beta, \alpha) .
\end{aligned}
$$

By (2.13) and (2.15),

for any $k$-form $\beta$.

$$
\delta \delta \beta=0
$$

One may finally introduce a discrete version of the Hodge $*$ operation. Given a $k$-cell $c_{k} \subset \mathbb{Z}^{D}$, let $c_{D-k}^{*}$ denote the $(D-k)$-cell in the dual lattice $\left(\mathbb{Z}^{D}\right)^{*}$ passing through $c_{k}$ and with orientation chosen such that it matches the orientation of $c_{k}$. Given some $k$-form $\alpha$, we define a $(D-k)$-form $* \alpha$ by

$$
(* \alpha)\left(c_{D-k}^{*}\right)=\alpha\left(c_{k}\right) \text {. }
$$

It is easy to see that

$$
* d * \alpha=\delta \alpha
$$

For

We will need the following

$$
\begin{aligned}
(* d * \alpha)\left(c_{k-1}\right) & =(d * \alpha)\left(c_{D-k+1}^{*}\right) \\
& =\sum_{c_{D-k}^{*} \subset \partial c_{D-k+1}^{*}}(* \alpha)\left(c_{D-k}^{*}\right) \\
& =\sum_{c_{k}: \partial c_{k} \supset c_{k-1}} \alpha\left(c_{k}\right) \\
& =(\delta \alpha)\left(c_{k-1}\right) .
\end{aligned}
$$


Lemma 1 (Poincaré). Let $\alpha$ be a $k$-form with values in $K(=\mathbb{Z}, \mathbb{R}, \mathbb{C})$ such that $\delta \alpha=0$. Then there exists $a(k+1)$-form $\beta$ with values in $K$ such that

$$
\alpha=\delta \beta \text {. }
$$

Moreover $\beta$ can be chosen such that $\operatorname{supp} \beta$ is contained in the smallest hypercube $\Omega_{\alpha}$ containing $\operatorname{supp} \alpha$, and $\max \left|\beta\left(c_{k+1}\right)\right| \leqq \sum_{c_{k} \in \operatorname{supp} \alpha}^{\prime}\left|\alpha\left(c_{k}\right)\right|$.

Remark. Similar statements hold with $\delta$ replaced by $d$. They can be obtained from Lemma 1 by using the * operation. The proof of Lemma 1 is quite elementary and is not given here.

\subsection{Fourier Transformation}

Next, we calculate the Fourier transform of the measure $d \mu_{\beta}\left(\theta_{A}\right)$ introduced in (2.3), (2.4). Let $\hat{\varphi}_{\beta}(n)$ denote the $n^{\text {th }}$ Fourier coefficient of $\varphi_{\beta}(\theta)$. First, we reexpress the partition function. Using (2.15), we obtain

$$
\begin{aligned}
\hat{Z}_{\Lambda} & =\int \prod_{p \subset \Lambda} \varphi_{\beta}\left(d \theta_{p}\right) \prod_{x y \subset \Lambda} d \theta_{x y} \\
& =\int \prod_{p \subset A}\left\{\sum_{n_{p} \in \mathbb{Z}} \hat{\varphi}_{\beta}\left(n_{p}\right) e^{i n_{p}(d \theta)_{p}}\right\} \prod_{x y \subset \Lambda} d \theta_{x y} \\
& =\sum_{n=\left\{n_{p}\right\}_{p} \subset \Lambda} \prod_{p \subset A} \hat{\varphi}_{\beta}\left(n_{p}\right) \int_{x y \subset \Lambda} e^{i \theta_{x y}(\delta n)_{x y}} d \theta_{x y} \\
& =(2 \pi)^{L(\Lambda)} \sum_{n: \delta n=0} \prod_{p \subset \Lambda} \hat{\varphi}_{\beta}\left(n_{p}\right),
\end{aligned}
$$

where $L(\Lambda)$ is the number of links (oriented bonds) in $\Lambda$. For $\varphi_{\beta}$ as in (2.2),

$$
\hat{\varphi}_{\beta}(n)=c e^{-(1 / 2 \beta) n^{2}},
$$

for some positive constant $c$.

Thus

$$
\hat{Z}_{\Lambda}=(2 \pi)^{L(\Lambda)} c^{P(\Lambda)} Z_{\Lambda}
$$

where

$$
Z_{\Lambda}=\sum_{n: \delta n=0} \prod_{p \subset \Lambda} e^{-(1 / 2 \beta) n_{p}^{2}}
$$

and $P(\Lambda)$ is the number of plaquettes in $\Lambda$.

Since $\delta n=0$,

$$
n=\delta m,
$$

for some 3-form $m$, and the support of $m$ can be chosen to lie within $\Lambda$ (see Lemma 1). Now,

$$
m=* \alpha,
$$

where $\alpha$ is a 1 -form on $\Lambda^{*}$, the dual of $\Lambda$. Thus

$$
n=* d \alpha \text {. }
$$


We note that $\alpha$ is not uniquely determined by $n$ : If $n=* d \alpha^{\prime}$ then $\alpha^{\prime}=\alpha+d \gamma$, for some scalar function $\gamma$ on $\left(\mathbb{Z}^{4}\right)^{*}$. Next, using (2.24)

$$
\begin{aligned}
(n, n)=\sum_{p \subset \Lambda} n_{p}^{2} & =\sum_{p \subset \Lambda}(* d \alpha)_{p}^{2}=\sum_{p \subset \Lambda}(d \alpha)_{p^{*}}^{2} \\
& =(d \alpha, d \alpha)_{\Lambda^{*}},
\end{aligned}
$$

where $p^{*}$ is the plaquette dual to $p$. Hence

$$
\begin{aligned}
Z_{\Lambda} & =\sum_{n: \delta n=0} e^{-(1 / 2 \beta)(n, n)} \\
& =\sum_{[\alpha]: \alpha_{\lambda y} \in \mathbb{Z}} e^{-(1 / 2 \beta)(d \alpha, d \alpha)},
\end{aligned}
$$

where $[\alpha]$ denotes the equivalence class $\left\{\alpha^{\prime}: \alpha^{\prime}=\alpha+d \gamma, \operatorname{supp} d \gamma \subset \Lambda^{*}\right\}$, and $\sum_{[\alpha]}$ indicates that only one configuration per equivalence class is retained in the summation.

Next, we compute the Fourier (duality) transform of $\langle W(\mathscr{L})\rangle_{\Lambda}(\beta)$. Let $\Sigma$ be the rectangle in the $0-1$-lattice plane whose boundary is the loop $\mathscr{L}$. The discrete version of Stokes' theorem says

$$
W(\mathscr{L})=\prod_{x y \subset \mathscr{L}} e^{i \theta_{x y}}=\prod_{p \subset \Sigma} e^{i(d \theta)_{p}},
$$

where all plaquettes $p \subset \Sigma$ have the same orientation as $\mathscr{L}$. Thus

$$
\langle W(\mathscr{L})\rangle_{\Lambda}(\beta)=\hat{Z}_{\boldsymbol{A}}^{-1} \int \prod_{p \subset \Lambda} \varphi_{\beta}\left(d \theta_{p}\right) \prod_{p \subset \Sigma} e^{i(d \theta)_{p}} \prod_{x y \subset \Lambda} d \theta_{x y} .
$$

The $n^{\text {th }}$ Fourier coefficient of $\varphi_{\beta}(\theta) e^{i \theta}$ is $\hat{\varphi}_{\beta}(n-1)$. Thus, as in (2.19), we obtain

$$
\begin{aligned}
\langle W(\mathscr{L})\rangle_{\Lambda}(\beta) & =\hat{Z}_{\Lambda}^{-1}(2 \pi)^{L(\Lambda)}\left\{\sum_{n: \delta n=0} \prod_{p \subset \Lambda \backslash \Sigma} \hat{\varphi}\left(n_{p}\right) \prod_{p \subset \Sigma} \hat{\varphi}\left(n_{p}-1\right)\right\} \\
& =Z_{\Lambda}^{-1}\left\{\sum_{n: \delta n=0} \prod_{p \subset \Lambda} e^{-(1 / 2 \beta) n_{p}^{2}} \prod_{p \subset \Sigma} e^{(1 / \beta) n_{p}-1 / 2 \beta}\right\} .
\end{aligned}
$$

As in (2.23)-(2.26),

$$
\begin{aligned}
\langle W(\mathscr{L})\rangle_{\Lambda}(\beta) & =\int d \mu_{\Lambda}(\alpha) D_{\partial \Sigma}(\alpha) \\
& \equiv\left\langle D_{\partial \Sigma}\right\rangle_{\Lambda}^{*}(\beta),
\end{aligned}
$$

where $d \mu_{\Lambda}(\alpha)$ is the discrete measure on the space of equivalence classes, $[\alpha]$, which assigns to $[\alpha]$ the weight

$$
Z_{A}^{-1} e^{-(1 / 2 \beta)(d \alpha, d \alpha)_{\Lambda *}},
$$

$\langle-\rangle_{A}^{*}(\beta)$ denotes expectations in this measure, and $D_{\hat{\partial} \Sigma}$ is the disorder operator defined by

$$
D_{\partial \Sigma}(\alpha)=\prod_{p \subset \Sigma} e^{(1 / \beta)(d \alpha)_{p \star}} e^{-1 / 2 \beta} .
$$

More generally,

for any choice of $\varphi_{\beta}$.

$$
\langle W(\mathscr{L})\rangle_{\Lambda}(\beta)=\left\langle\prod_{p \subset \Sigma} \frac{\hat{\varphi}_{\beta}\left((d \alpha)_{p^{*}}-1\right)}{\hat{\varphi}_{\beta}\left((d \alpha)_{p^{*}}\right)}\right\rangle_{\Lambda}^{*}(\beta),
$$




\subsection{The Gaussian U(1) Lattice Model}

Consider the (infrared) regularized Gaussian measure

$$
d \mu_{\Lambda, \varepsilon}^{0}(\alpha)=N_{\Lambda, \varepsilon}^{-1} e^{-(1 / 2 \beta)\left\{(d \alpha, d \alpha)_{\Lambda^{*}}+\varepsilon(\alpha, \alpha)_{\Lambda *}\right\}} \prod_{x y \subset \Lambda^{*}} d \alpha_{x y},
$$

where $d \alpha_{x y}$ is the Lebesgue measure on $\mathbb{R}, \alpha_{x y}=0$, for $x y \not \subset \Lambda^{*}, \varepsilon>0$ is an (infrared) regulator mass, and $N_{\Lambda, \varepsilon}$ is a normalization factor chosen such that $\int d \mu_{\Lambda, \varepsilon}^{0}(\alpha)=1$. Let $\Pi_{\Lambda^{*}}$ denote the orthogonal [with respect to $(\cdot, \cdot)$ ] projection onto the space of 1 -forms with support in $\Lambda^{*}$. Let $V_{\Lambda, \varepsilon}$ be the inverse, on the space of 1-forms with support in $\Lambda^{*}$, of $\Pi_{\Lambda^{*}}(\delta d+\varepsilon)$. Clearly, $d \mu_{\Lambda, \varepsilon}^{0}$ is the Gaussian measure with mean zero and covariance $V_{\Lambda, \varepsilon}$. Thus

$$
\int d \mu_{\Lambda, \varepsilon}^{0}(\alpha) e^{i \alpha(\mu)}=e^{-(\beta / 2)\left(\mu, V_{\Lambda, \varepsilon} \mu\right)_{\Lambda *}},
$$

for any 1 -form $\mu$ with $\operatorname{supp} \mu \subseteq \Lambda^{*}$. Here $\alpha(\mu) \equiv(\alpha, \mu)_{\Lambda^{*}}=\sum_{x y \subset \Lambda^{*}} \alpha_{x y} \mu_{x y}$.

[Note if $\Lambda^{*}=\left(\mathbb{Z}^{4}\right)^{*}$

$$
V_{\varepsilon}=\left(1+\varepsilon^{-1} d \delta\right)(-\Delta+\varepsilon)^{-1},
$$

where $-\Delta=d \delta+\delta d$, because $(\delta d+\varepsilon)\left(1+\varepsilon^{-1} d \delta\right)=-\Delta+\varepsilon+\varepsilon^{-1} \delta d d \delta=-\Delta+\varepsilon$, by (2.13).]

When $\varepsilon$ tends to 0 the right side of (2.33) tends to 0 on all of those 1 -forms $\mu$ with supp $\mu \subseteq \Lambda^{*}$ and $(d \mu, d \mu)_{\Lambda^{*}}=0$, i.e. $\mu=d v$, for some function $v$. Since $\{\mu: \delta \mu=0$, $\left.\operatorname{supp} \mu \subseteq \Lambda^{*}\right\}$ is orthogonal to $\left\{\mu: d \mu=0, \operatorname{supp} \mu \subseteq \Lambda^{*}\right\}$,

$$
\lim _{\varepsilon \downarrow 0} \int d \mu_{\Lambda, \varepsilon}^{0}(\alpha) e^{i \alpha(\mu)}= \begin{cases}e^{-(\beta / 2)\left(\mu, V_{\Lambda} \mu\right)_{\Lambda^{*}}}, & \text { if } \delta \mu=0 \\ 0, & \text { otherwise. }\end{cases}
$$

Here $V_{A}$ is the inverse, on the space of 1 -forms $\left\{\mu: \delta \mu=0\right.$, supp $\left.\mu \cong \Lambda^{*}\right\}$, of $\Pi_{\Lambda^{*}} \delta d$. On that subspace

$$
\Pi_{\Lambda^{*}} \delta d \mu=\Pi_{\Lambda^{*}}(d \delta+\delta d) \mu \equiv-\Delta_{\Lambda} \mu,
$$

where $\Delta_{A}$ is the finite difference Laplacian with 0 Dirichlet data on the outer boundary of $\Lambda^{*}$, so that

$$
\left(\mu, V_{\Lambda} \mu\right)_{\Lambda^{*}}=\left(\mu,\left(-\Delta_{\Lambda}\right)^{-1} \mu\right)_{\Lambda^{*}} \quad \text { if } \quad \delta \mu=0 .
$$

We denote by $d \mu_{\Lambda}^{0}(\alpha)$ the measure on the space of equivalence classes, $[\alpha]=\left\{\alpha^{\prime}: d \alpha^{\prime}=d \alpha\right\}$, determined by

$$
\int d \mu_{\Lambda}^{0}(\alpha) e^{i \alpha(\mu)}=e^{-(\beta / 2)\left(\mu,\left(-\Delta_{\Lambda}\right)^{-1} \mu\right)_{\Lambda *}}
$$

for all 1-forms $\mu$, with $\delta \mu=0$.

\subsection{The Poisson Summation}

We now reexpress the discrete measure $d \mu_{\Lambda}$ introduced in (2.28), (2.29) in terms of $d \mu_{A}^{0}$ by inserting the constraints

$$
\alpha_{x y} \in \mathbb{Z}, \text { for all } x y \subset \Lambda^{*}: d \mu_{\Lambda}(\alpha)=\Xi_{\Lambda}^{-1} \prod_{x y \subset \Lambda^{*}}\left\{\sum_{q_{x y}^{\prime} \in \mathbb{Z}} \delta\left(\alpha_{x y}-q_{x y}^{\prime}\right)\right\} d \mu_{\Lambda}^{0}(\alpha),
$$


where $\Xi_{\Lambda}$ is the normalization factor for which $\int d \mu_{\Lambda}(\alpha)=1$. We now apply the Poisson summation formula:

$$
\sum_{q_{x y}^{\prime} \in \mathbb{Z}} \delta\left(\alpha_{x y}-q_{x y}^{\prime}\right)=1+2 \sum_{(2 \pi)^{-1} q_{x y}=1}^{\infty} \cos \left(q_{x y} \alpha_{x y}\right) .
$$

Let $\left\{z_{q}\right\}_{q=1}^{\infty}$ be a sequence of numbers such that $2 \sum_{(2 \pi)^{-1} q=1}^{\infty} z_{q}^{-1}=1$. (A specific such sequence will be chosen later.) Then

$$
1+2 \sum_{(2 \pi)^{-1} q_{x y}=1}^{\infty} \cos \left(q_{x y} \alpha_{x y}\right)=\sum_{(2 \pi)^{-1} q_{x y}=1}^{\infty} 2 z_{q_{x y}}^{-1}\left(1+z_{q_{x y}} \cos \left(q_{x y} \alpha_{x y}\right)\right) .
$$

Let

$$
q \equiv\left\{q_{x y}\right\}_{x y \subset \Lambda^{*}}, \quad c_{q} \equiv \prod_{x y \subset \Lambda^{*}} 2 z_{q_{x y}}^{-1}
$$

By (2.37) and (2.38)

$$
\Xi_{\Lambda} d \mu_{\Lambda}(\alpha)=\sum_{q} c_{q} \prod_{x y \subset \Lambda^{*}}\left(1+z_{q_{x y}} \cos \left(q_{x y} \alpha_{x y}\right)\right) d \mu_{\Lambda}^{0}(\alpha) .
$$

We now need some definitions:

A current distribution (or density) $\varrho$ is a mapping from the set $\mathscr{B}$ of directed bonds (links) to $2 \pi \mathbb{Z}$, of finite support. An ensemble $\mathscr{E}$ is a family of current densities, $\varrho$, with the properties that

$$
\begin{gathered}
\operatorname{supp} \varrho \subseteq \Lambda^{*}, \text { for all } \varrho \in \mathscr{E} \\
\text { supp } \varrho \cap \operatorname{supp} \varrho^{\prime}=\emptyset, \text { for all } \varrho \text { and } \varrho^{\prime} \text { in } \mathscr{E} \text { with } \varrho \neq \varrho^{\prime} .
\end{gathered}
$$

A $k$-ensemble, $\mathscr{E}^{k}$, is an ensemble with the property that

$$
\operatorname{dist}\left(\varrho, \varrho^{\prime}\right) \geqq 2^{k / 2}, \quad k=0,1,2, \ldots,
$$

where $\operatorname{dist}\left(\varrho, \varrho^{\prime}\right)$ denotes the Euclidean distance between supp $\varrho$ and $\operatorname{supp} \varrho^{\prime}$. Finally, let $\alpha(\varrho) \equiv \sum_{x y}^{\prime} \alpha_{x y} \varrho_{x y}$.

\section{Lemma 2.}

$$
\prod_{x y \subset A^{*}}\left(1+z_{q_{x y}} \cos \left(q_{x y} \alpha_{x y}\right)\right)=\sum_{\gamma} c_{\gamma} \prod_{\varrho \in \mathscr{\sigma}_{\gamma}^{\prime}}[1+K(\varrho) \cos (\alpha(\varrho))],
$$

where $\gamma$ ranges over some finite index set, each $\mathscr{E}_{\gamma}^{1}$ is a 1-ensemble and

ii)

$$
\begin{gathered}
c_{\gamma}>0, \text { for all } \gamma \\
0<K(\varrho) \leqq 3^{N_{1}(\operatorname{supp} \varrho)} \prod_{x y(\operatorname{supp} \varrho} z_{\left|\varrho_{x y}\right|},
\end{gathered}
$$

where $N_{1}(\operatorname{supp} \varrho)$ is the number of links within distance $\leqq 1$ of the support of $\varrho$.

Proof. Lemma 2 is a simple special case of Lemma 2.2 in [15]. For this reason we only present a sketch of the proof. (The reader will find it easy to supply the 
details.) The proof follows by successive applications of the identity

$$
\begin{aligned}
{[1+} & \left.K_{1} \cos \left(\alpha\left(\varrho_{1}\right)\right)\right]\left[1+K_{2} \cos \left(\alpha\left(\varrho_{2}\right)\right)\right] \\
= & 1 / 3\left[1+3 K_{1} \cos \left(\alpha\left(\varrho_{1}\right)\right)\right]+1 / 3\left[1+3 K_{2} \cos \left(\alpha\left(\varrho_{2}\right)\right)\right] \\
& +1 / 6\left[1+3 K_{1} K_{2} \cos \left(\alpha\left(\varrho_{1}-\varrho_{2}\right)\right)\right] \\
& +1 / 6\left[1+3 K_{1} K_{2} \cos \left(\alpha\left(\varrho_{1}+\varrho_{2}\right)\right)\right] .
\end{aligned}
$$

First (2.42) is applied to any two factors,

$$
\left(1+z_{q_{x y}} \cos \left(q_{x y} \alpha_{x y}\right)\right), \quad\left(1+z_{q_{x^{\prime} y^{\prime}}} \cos \left(q_{x^{\prime} y^{\prime}}, \alpha_{x^{\prime} y^{\prime}}\right)\right),
$$

in

$$
I_{\Lambda^{*}} \equiv \prod_{x y \subset \Lambda^{*}}\left(1+z_{q_{x y}} \cos \left(q_{x y} \alpha_{x y}\right)\right)
$$

for which $\operatorname{dist}\left(x y, x^{\prime} y^{\prime}\right)=0$. The right side of (2.42) is, for each such pair of factors, inserted in $I_{\Lambda^{*}}$, and the result is expanded as a sum of products. After a finite number of such expansion steps one obtains

$$
I_{\Lambda^{*}}=\sum_{\lambda} c_{\lambda} \prod_{\varrho \in \mathscr{E}_{\lambda}}\left[1+K^{\prime}(\varrho) \cos (\alpha(\varrho))\right]
$$

where $\left\{\mathscr{E}_{\lambda}\right\}$ is some family of ensembles, and by (2.42) each $c_{\lambda}$ is the product of a power of $1 / 3$ and a power of $1 / 6$. If all $\mathscr{E}_{\lambda}$ are 1 -ensembles, no further applications of (2.42) are necessary, and (2.41) is proven.

If however some ensembles $\mathscr{E}_{\lambda_{1}}, \mathscr{E}_{\lambda_{2}}, \ldots$ are not 1 -ensembles, yet, one applies (2.42) to any pair of factors $\left[1+K^{\prime}\left(\varrho_{1}\right) \cos \left(\alpha\left(\varrho_{1}\right)\right)\right],\left[1+K^{\prime}\left(\varrho_{2}\right) \cos \left(\alpha\left(\varrho_{2}\right)\right)\right]$, with the property that $\varrho_{1}, \varrho_{2}$ are in $\mathscr{E}_{\lambda_{i}}$, for some $i$, and $\operatorname{dist}\left(\varrho_{1}, \varrho_{2}\right) \leqq 1$, the right side of $(2.42)$ is inserted on the right side of (2.44) and expanded as a sum of products for all $i=1,2, \ldots$. Since $\Lambda^{*}$ is finite, the combinatorial expansion described here terminates after finitely many applications of (2.42), (when all resulting ensembles are 1-ensembles), and (2.41) follows.

We now check i) and ii) in Lemma 2. If a current density $\varrho$ has been obtained by pairing $\varrho_{1}$ and $\varrho_{2}$, in the sense of identity (2.42), e.g. $\varrho=\varrho_{1} \pm \varrho_{2}$, then

$$
K(\varrho)=3 K\left(\varrho_{1}\right) K\left(\varrho_{2}\right) .
$$

If $\varrho=\varrho_{\alpha}, \alpha=1,2$, i.e. one of the first two terms on the right side of $(2.42)$ has been retained,

$$
K\left(\varrho_{\alpha}\right) \rightarrow K(\varrho)=3 K\left(\varrho_{\alpha}\right) .
$$

Thus, given some $\varrho \in \mathscr{E}_{\gamma}^{1}$, for some $\gamma$, one easily verifies that

$$
K(\varrho)=3^{n(\varrho)} \prod_{x y \subset \text { supp } \varrho} z_{\left|\varrho_{x y}\right|}
$$

where $n(\varrho)$ is the number of applications of $(2.42)$ that were necessary to obtain $\varrho$. A minute of reflection shows that

$$
n(\varrho) \leqq N_{1}(\operatorname{supp} \varrho),
$$

which establishes ii) (see also Sect. 2 of [15]). Finally, $c_{\gamma}$ is clearly of the form

$$
c_{\gamma}=(1 / 3)^{n_{\gamma}}(1 / 6)^{m_{\gamma}},
$$


where $n_{\gamma}$ and $m_{\gamma}$ are the following positive integers: The total number of times (2.42) has been applied in the inductive construction of $\mathscr{E}_{\gamma}^{1}$ is $n_{\gamma}+m_{\gamma}$, and $n_{\gamma}$ times one of the first two terms on the right side of (2.42) has been retained, whereas $m_{\gamma}$ times one of the second two terms has been retained. This yields i).

Remarks. 1) Combining (2.40) and (2.41) one obtains

$$
\Xi_{\Lambda} \cdot d \mu_{\Lambda}(\alpha)=\sum_{\gamma} d_{\gamma} \prod_{\varrho \in \mathscr{N}_{\gamma}^{1}}[1+K(\varrho) \cos (\alpha(\varrho))] d \mu_{\Lambda}^{0}(\alpha)
$$

where $\left\{\mathscr{N}_{\gamma}^{1}\right\}$ is a family of 1 -ensembles, and $d_{\gamma}>0$, for all $\gamma$. Moreover, $K(\varrho)$ still satisfies ii) of Lemma 2.

Since any two current densities $\varrho_{1}$ and $\varrho_{2} \neq \varrho_{1}$ in some $\mathscr{N}_{\gamma}^{1}$ satisfy $\operatorname{dist}\left(\varrho_{1}, \varrho_{2}\right) \geqq \sqrt{2}$, we conclude that, for each subensemble $\mathscr{E}_{\gamma}^{1} \cong \mathscr{N}_{\gamma}^{1}$,

$$
\int \prod_{\varrho \in \mathscr{E}_{\gamma}^{1}} K(\varrho) \cos (\alpha(\varrho)) d \mu_{\Lambda}^{0}(\alpha)=0
$$

unless $\delta \varrho=0$, for all $\varrho \in \mathscr{E}_{\gamma}^{1}$, for all $\gamma$. This follows from (2.34) and (2.36). Thus all factors on the right side of (2.46) labelled by some current density $\varrho$ for which $\delta \varrho \neq 0$ may be omitted. Therefore

$$
\Xi_{\Lambda} \cdot d \mu_{\Lambda}(\alpha)=\sum_{\gamma} d_{\gamma} \prod_{\substack{\varrho \in \mathcal{N}_{\gamma}^{1} \\ \delta \varrho=0}}[1+K(\varrho) \cos (\alpha(\varrho))] d \mu_{\Lambda}^{0}(\alpha) .
$$

2) For the study of more general lattice gauge theories it is interesting to note that Lemma 2 can be generalized by replacing 1 -ensembles by $k$-ensembles, $k=2,3, \ldots$, on the right side of $(2.41)$. In ii) the exponent $N_{1}$ (supp @) must then be replaced by a quantity $N_{k}(\operatorname{supp} \varrho)$, the definition of which along with upper bounds can easily be inferred from Theorem 2.1 and Lemma 2.2 of [15]. The resulting combinatorial scheme can be used, for example, to give a simple, new form of the high and (in the discrete case) low temperature expansion for the expectation of the Wilson (or disorder) loop in lattice gauge theories with interactions of finite range. This permits us to prove, in particular, that any pure lattice gauge theory with a discrete (abelian or non-abelian) gauge group and interaction of finite range does not confine static quarks if $\beta$ is large enough. This extends the result in [2].

\subsection{A Change of Variables}

Our purpose is now to start estimating

$$
\langle W(\mathscr{L})\rangle_{\Lambda}(\beta)=\int d \mu_{\Lambda}(\alpha) D_{\partial \Sigma}(\alpha),
$$

see $2.4,(2.28)-(2.31)$, by making use of Eq. (2.47) for $d \mu_{\Lambda}(\alpha)$ and changing variables

$$
\alpha \rightarrow \alpha+\tau,
$$

where $\tau$ is a 1 -form defined as follows: Let $\sigma$ be the 2-form given by

$$
\sigma\left(p^{*}\right)= \begin{cases}1, & p=\left(p^{*}\right)^{*} \in \Sigma \\ 0, & \text { otherwise }\end{cases}
$$


where $\Sigma$ is the rectangle defined in 2.4 , with $\partial \Sigma=\mathscr{L}$. [If $\Lambda$ is large enough, $\Sigma \subset \Lambda$ and $\operatorname{dist}(\Sigma, \partial \Lambda)>0$.] We set

$$
\tau=-\delta \Delta_{\Lambda}^{-1} \sigma,
$$

where $\Delta_{A}$ is the finite difference Laplacian with 0 Dirichlet data on the outer boundary of $\Lambda^{*}$ introduced in 2.5 . Clearly

with

$$
\begin{aligned}
\Pi_{\Lambda^{*}} d \tau & =-\Pi_{\Lambda^{*}} d \delta \Delta_{\Lambda}^{-1} \sigma=-(d \delta+\delta d)_{\Lambda} \Delta_{\Lambda}^{-1} \sigma+\Pi_{\Lambda^{*}} \delta d \Delta_{\Lambda}^{-1} \sigma=\sigma-\varepsilon_{\Lambda}, \\
\varepsilon_{\Lambda} & \equiv-\Pi_{\Lambda^{*}} \delta d \Delta_{\Lambda}^{-1} \sigma .
\end{aligned}
$$

Under this change of variables

$$
\begin{gathered}
d \mu_{\Lambda}^{0}(\alpha) \rightarrow d \mu_{\Lambda}^{0}(\alpha) e^{-(1 / \beta)(d \alpha, d \tau)_{\Lambda^{*}}} e^{-(1 / 2 \beta)(d \tau, d \tau)_{\Lambda^{*}}} \\
=d \mu_{\Lambda}^{0}(\alpha) \prod_{p \in \Sigma} e^{-(1 / \beta)(d \alpha)_{p^{*}}} e^{-1 / 2 \beta} \\
\cdot e^{(1 / \beta)\left(\sigma, \varepsilon_{\Lambda}\right)} e^{-(1 / 2 \beta)\left(\varepsilon_{\Lambda}, \varepsilon_{\Lambda}\right)}
\end{gathered}
$$

This follows from the definition of $d \mu_{\Lambda}^{0}(\alpha)$, see 2.5, (2.32)-(2.34) and of $\tau$ by using the fact that $\left(d \alpha, \varepsilon_{\Lambda}\right)_{\Lambda^{*}}=0$.

By (2.30)

$$
\begin{gathered}
D_{\partial \Sigma}(\alpha) \rightarrow \prod_{p \in \Sigma}\left\{e^{(1 / \beta)(d \alpha)_{p^{*}}} e^{-1 / 2 \beta} e^{(1 / \beta)(d \tau) p_{p^{*}}}\right\} \\
=e^{-(1 / \beta)\left(\sigma, \varepsilon_{A}\right)} \prod_{p \in \Sigma} e^{(1 / \beta)(d \alpha)_{p^{*}}} e^{1 / 2 \beta}
\end{gathered}
$$

Combining (2.52) and (2.53) we get

$$
D_{\partial \Sigma}(\alpha) d \mu_{\Lambda}^{0}(\alpha) \rightarrow e^{-(1 / 2 \beta)\left(\varepsilon_{\Lambda}, \varepsilon_{\Lambda}\right)} d \mu_{\Lambda}^{0}(\alpha) .
$$

Finally,

$$
\prod_{\substack{\varrho \in \mathscr{N}^{\frac{1}{\gamma}} \\ \delta \varrho=0}}[1+K(\varrho) \cos (\alpha(\varrho))] \rightarrow \prod_{\substack{\varrho \in \mathcal{N}^{\frac{1}{\gamma}} \\ \delta \varrho=0}}[1+K(\varrho) \cos (\alpha(\varrho)+\tau(\varrho))] .
$$

Since $\delta \varrho=0, \varrho=\delta \mu_{\varrho}$, where $\mu_{\varrho}$ is a 2 -form with $\mu_{\varrho}\left(p^{*}\right) \in 2 \pi \mathbb{Z}$, for all $p^{*} \subset \Lambda^{*}$, and $\operatorname{supp} \mu_{\varrho} \subseteq \Omega_{\varrho} \subseteq \Lambda^{*}$ (see 2.3, Lemma 1). Thus, using (2.51) we see that

$$
\tau(\varrho)=\left(d \tau, \mu_{\varrho}\right)_{\Lambda^{*}}=\left(\sigma, \mu_{\varrho}\right)-\left(\varepsilon_{\Lambda}, \mu_{\varrho}\right)_{\Lambda^{*}},
$$

so that by (2.49) and the periodicity of the cosine

$$
\cos (\alpha(\varrho)+\tau(\varrho))=\cos \left(\alpha(\varrho)-\left(\varepsilon_{\Lambda}, \mu_{\varrho}\right)_{\Lambda^{*}}\right) \text {. }
$$

Combining representation (2.47) of $d \mu_{\Lambda}(\alpha)$ with (2.54)-(2.57) we obtain

$$
\begin{aligned}
\langle W(\mathscr{L})\rangle_{\Lambda}(\beta)= & \Xi_{\Lambda}^{-1} e^{-(1 / 2 \beta)\left(\varepsilon_{\Lambda}, \varepsilon_{\Lambda}\right)} \sum_{\gamma} d_{\gamma} \\
& \cdot \int \prod_{\substack{\varrho \in \mathscr{N}^{\frac{1}{\gamma}} \\
\delta \varrho=0}}\left[1+K(\varrho) \cos \left(\alpha(\varrho)-\left(\varepsilon_{\Lambda}, \mu_{\varrho}\right)_{\Lambda^{*}}\right)\right] d \mu_{\Lambda}^{0}(\alpha),
\end{aligned}
$$

where

$$
\Xi_{\Lambda}=\sum_{\gamma} d_{\gamma} \int \prod_{\substack{\varrho \in \mathcal{N}_{\gamma}^{1} \\ \delta \varrho=0}}[1+K(\varrho) \cos (\alpha(\varrho))] d \mu_{\Lambda}^{0}(\alpha)
$$




\subsection{The Renormalization Transformation}

In this section we propose to renormalize the current densities $\varrho, \varrho \rightarrow \bar{\varrho}$, and activities $K(\varrho), K(\varrho) \rightarrow z(\beta, \varrho)$, in such a way that

$$
\begin{aligned}
\int & \prod_{\substack{\varrho \in \mathscr{N}^{1} \\
\delta}}\left[1+K(\varrho) \cos \left(\alpha(\varrho)-\theta_{\varrho}\right)\right] d \mu_{\Lambda}^{0}(\alpha) \\
& =\int \prod_{\substack{\varrho \in \mathcal{N}_{\mathcal{Y}}^{1} \\
\delta \varrho=0}}\left[1+z(\beta, \bar{\varrho}) \cos \left(\alpha(\bar{\varrho})-\theta_{\varrho}\right)\right] d \mu_{\Lambda}^{0}(\alpha),
\end{aligned}
$$

with $z(\beta, \bar{\varrho}) \ll 1$, for $\beta$ sufficiently large. Here $\theta_{\varrho}\left(=0\right.$ or $\left.\left(\varepsilon_{\Lambda}, \mu_{\varrho}\right)_{\Lambda^{*}}\right)$ are real phases. Given some current density $\varrho \in \mathscr{N}_{\gamma}^{1}$, it is easy to see that we can choose a subset $\mathscr{B}_{\varrho}$ of links in supp $\varrho$ with the property that two different links in $\mathscr{B}_{\varrho}$ do not belong to a common plaquette and that

$$
\sum_{x y \in \mathscr{B} \varrho}\left|\varrho_{x y}\right|^{2} \geqq c\|\varrho\|_{2}^{2},
$$

where $\|\varrho\|_{p}^{p} \equiv \sum_{x y}\left|\varrho_{x y}\right|^{p}, p=1,2,3, \ldots$, and $c$ is a purely geometrical constant, namely

$$
\begin{aligned}
c^{-1} & =\operatorname{card}\left\{b^{\prime}: b^{\prime} \neq b, b^{\prime} \in \partial p \text { for some } p \text { with } \partial p \ni b\right\} \\
& =18, \text { in four dimensions. }
\end{aligned}
$$

Since $\operatorname{dist}\left(\varrho_{1}, \varrho_{2}\right) \geqq \sqrt{2}$, for two current densities $\varrho_{1}$ and $\varrho_{2} \neq \varrho_{1}$ in some ensemble $\mathscr{N}_{\gamma}^{1}$, the choice of $\mathscr{B}_{\varrho}$, for a given current density $\varrho \in \mathscr{N}_{\gamma}^{1}$, can be made independently of all other current densities in $\mathscr{N}_{\gamma}^{1}$ in such a way that (2.61) holds.

Our renormalization transformation is based on the following simple identity

Lemma 3. Let $x y \subset \Lambda^{*}$, and let $G(\alpha)$ be a function which does not depend on $\alpha_{x y}$. Then

$$
\int e^{i \varrho \alpha_{x y}} G(\alpha) d \mu_{\Lambda}^{0}(\alpha)=e^{-\left(\beta / 2 n_{x y}\right) \varrho^{2}} \cdot \int e^{-i \varrho \bar{\alpha}_{x y}} G(\alpha) d \mu^{0}(\alpha),
$$

where

$$
\bar{\alpha}_{x y}=\left(1 / n_{x y}\right)(\delta d \alpha)_{x y}-\alpha_{x y},
$$

and

$$
\begin{aligned}
n_{x y} & =\operatorname{card}\left\{p^{*}: p^{*} \subset \Lambda^{*}, \partial p^{*} \ni x y\right\} \\
& =6, \text { in dimension } 4
\end{aligned}
$$

(unless $x y$ belongs to the boundary of $\Lambda^{*}$ ).

Remark. It is important to note that $\bar{\alpha}_{x y}$ is independent of $\alpha_{x y}$ and that $n_{x y} \leqq 6$, so that

$$
e^{-\left(\beta / n_{x y}\right) \varrho^{2}} \leqq e^{-(\beta / 6) \varrho^{2}}
$$

Proof. In the following, all formal calculations hold rigorously if $d \mu_{\Lambda}^{0}(\alpha)$ is first replaced by $d \mu_{\Lambda, \varepsilon}^{0}(\alpha)$. Since the existence of the limit $\varepsilon \downarrow 0$ does not pose any problem (for finite $\Lambda$ ), that regularization is omitted right away. 
Our proof relies on explicitly integrating over $\alpha_{x y}$, using the following obvious equation for $d \mu_{\Lambda}^{0}(\alpha)$ :

$$
d \mu_{\Lambda}^{0}(\alpha)=d \varrho_{\Lambda \sim(x y)}(\alpha) \prod_{\substack{p^{*} \\ x y \in \partial p^{*} \subset}} e^{-(1 / 2 \beta)(d \alpha)_{p^{*}}^{2}} d \alpha_{x y},
$$

where $d \varrho_{\Lambda \sim(x y)}(\alpha)$ is a finite measure independent of $\alpha_{x y}$. By changing variables,

$$
\alpha_{x y} \mapsto \alpha_{x y}+i\left(\beta / n_{x y}\right) \varrho,
$$

we obtain

$$
\begin{aligned}
\int & \prod_{\partial p^{*} \ni x y} e^{-(1 / 2 \beta)(d \alpha)_{p^{\star}}^{2}} e^{i \varrho \alpha_{x y}} d \alpha_{x y} \\
& =\int \prod_{\partial p^{*} \ni x y} e^{-(1 / 2 \beta)\left((d \alpha)_{p^{\star}}+i\left(\beta / n_{x y}\right) \varrho^{2}\right.} e^{i \varrho \alpha_{x y}} e^{-\left(\beta / n_{x y}\right) \varrho^{2}} d \alpha_{x y} \\
& =e^{-\left(\beta / 2 n_{x y}\right) \varrho^{2}} e^{-i \bar{x}_{\bar{x}} \varrho\left[(\delta d \alpha)_{x y}-n_{x y} \alpha_{x y}\right]} \int \prod_{\partial p^{*} \ni x y} e^{-(1 / 2 \beta)(d \alpha)_{p^{\star}}^{2}} d \alpha_{x y} .
\end{aligned}
$$

By combining (2.65) and (2.66) we obtain

$$
\int e^{i \varrho \alpha_{x y}} G(\alpha) d \mu_{\Lambda}^{0}(\alpha)=e^{-\left(\beta / 2 n_{x y}\right) \varrho 2} \int e^{-i \varrho \alpha_{x y}} G(\alpha) d \mu_{\Lambda}^{0}(\alpha) .
$$

We set $\sim \mathscr{B}_{\varrho} \equiv \operatorname{supp} \varrho \sim \mathscr{B}_{\varrho}$ and define a renormalized current density $\bar{\varrho}$ by the equation

$$
\begin{aligned}
\alpha(\bar{\varrho}) & =\sum_{x y \in \sim \mathscr{B}_{e}} \alpha_{x y} \bar{\varrho}_{x y} \\
& \equiv \sum_{x y \in \mathscr{B}_{\varrho}} \bar{\alpha}_{x y} \varrho_{x y}+\sum_{x y \in \sim \mathscr{B}_{\varrho}} \alpha_{x y} \varrho_{x y}
\end{aligned}
$$

for an arbitrary 1 -form $\alpha$, with $\operatorname{supp} \alpha \subseteq \Lambda^{*}$. Furthermore,

$$
z(\beta, \bar{\varrho})=K(\varrho) \exp \left[-(\beta / 2) \sum_{x y \in \mathscr{B}_{Q}} n_{x y}^{-1} \varrho_{x y}^{2}\right]
$$

By (2.61), (2.62), and (2.64)

$$
z(\beta, \bar{\varrho}) \leqq K(\varrho) \exp \left[-(\beta / 216)\|\varrho\|_{2}^{2}\right] .
$$

\section{Corollary 4.}

$$
\begin{aligned}
\Xi_{\Lambda}= & \sum_{\gamma} d_{\gamma} \int \prod_{\substack{\varrho \in \mathcal{N}^{1} \\
\delta \varrho=0}}[1+z(\beta, \bar{\varrho}) \cos (\alpha(\bar{\varrho}))] d \mu_{\Lambda}^{0}(\alpha), \\
\langle W(\mathscr{L})\rangle_{\Lambda}(\beta)= & \Xi_{\Lambda}^{-1} e^{-(1 / 2 \beta)\left(\varepsilon_{\Lambda}, \varepsilon_{\Lambda}\right)} \sum_{\gamma} d_{\gamma} \int \prod_{\substack{\varrho \in \mathcal{N}_{\gamma}^{1} \\
\delta \varrho=0}}[1+z(\beta, \bar{\varrho}) \\
& \left.\cdot \cos \left(\alpha(\bar{\varrho})-\left(\varepsilon_{\Lambda}, \mu_{\varrho}\right)_{\Lambda^{*}}\right)\right] d \mu_{\Lambda}^{0}(\alpha) .
\end{aligned}
$$

Proof. We apply the following obvious identities:

$$
\cos \left(\alpha(\varrho)-\theta_{\varrho}\right)=\frac{1}{2} e^{i\left(\alpha(\varrho)-\theta_{\varrho}\right)}+\frac{1}{2} e^{-i\left(\alpha(\varrho)-\theta_{\varrho}\right)},
$$

whence

$$
\prod_{\varrho \in \mathcal{N}_{\gamma}^{1}}\left[1+K(\varrho) \cos \left(\alpha(\varrho)-\theta_{\varrho}\right)\right]=\sum_{\mathscr{E}_{\gamma}^{1} \subseteq \mathcal{N} \frac{1}{\gamma}\{\sigma(\varrho)= \pm 1\}_{\varrho \in \mathscr{E}_{\gamma}^{1}}} \prod_{\varrho \in \mathscr{E}_{\gamma}^{1}}(1 / 2) \mathrm{K}(\varrho) e^{i \sigma(\varrho)\left(\alpha(\varrho)-\theta_{\varrho}\right)},
$$


where the first sum extends over all subensembles $\mathscr{E}_{\gamma}^{1} \subseteq \mathscr{N}_{\gamma}^{1}$;

$$
e^{ \pm i\left(\alpha(\varrho)-\theta_{\varrho}\right)}=e^{\mp i \theta_{\varrho}} \prod_{x y \in \mathscr{B}_{Q}} e^{ \pm i \varrho_{x y} \alpha_{x y}} \prod_{x y \in \sim \mathscr{B}_{Q}} e^{ \pm i \varrho_{x y} \alpha_{x y}} .
$$

We then use Lemma 3 to successively integrate out

$$
\prod_{\varrho \in \mathscr{E}_{\gamma}^{1}} \prod_{x y \in \mathscr{B}_{\varrho}} e^{i \sigma(\varrho) \varrho_{x y} \alpha_{x y}},
$$

for all $\mathscr{E}_{\gamma}^{1} \subseteq \mathscr{N}_{\gamma}^{1}$ and all $\{\sigma(\varrho)\}$. Since $\operatorname{dist}\left(\varrho_{1}, \varrho_{2}\right) \geqq \sqrt{2}$ for arbitrary $\varrho_{1}, \varrho_{2}$ in $\mathscr{N}_{\gamma}^{1}$ with $\varrho_{1} \neq \varrho_{2}$, and by our definition of $\mathscr{B}_{\varrho}, \varrho \in \mathscr{N}_{\gamma}^{1}$, the hypotheses of Lemma 3 remain valid after an arbitrary number $n=0,1,2, \ldots$ of integrations. When all integrations in each term have been carried out the above identities are applied in reverse, with $\alpha(\bar{\varrho})$ replaced by $\alpha(\varrho)$ and $K(\bar{\varrho})$ replaced by $z(\beta, \bar{\varrho})$.

\subsection{Estimates on $z(\beta, \bar{\varrho})$}

We recall that

$$
0<K(\varrho) \leqq 3^{N_{1}(\operatorname{supp} \varrho)} \prod_{x y \subset \operatorname{supp} \varrho} z_{\left|\varrho_{x y}\right|}
$$

where $\left\{z_{q}\right\}$ is a sequence with the property that

$$
\sum_{(2 \pi)^{-1} q=1}^{\infty} z_{q}^{-1}=1 / 2
$$

see $2.6[(2.37),(2.38)$, and Lemma 2]. We now choose this sequence explicitly, for example as follows :

$$
z_{q}=e^{\beta_{0} q^{2}},
$$

where $\beta_{0}$ is that positive constant for which (2.72) holds. A simple, geometric estimate on $N_{1}(\operatorname{supp} \varrho)$ then yields

$$
0<K(\varrho) \leqq \sum_{x y \subset \operatorname{supp} \varrho} e^{\beta_{1}\left|\varrho_{x y}\right|^{2}}
$$

for some finite constant $\beta_{1}$.

Combining (2.74) with (2.70) we obtain

$$
0<z(\beta, \bar{\varrho}) \leqq \exp \left[\left(\beta_{1}-\beta / 216\right)\|\varrho\|_{2}^{2}\right] .
$$

Thus, if $\beta>216 \beta_{1}$ (a fairly large number, alas)

$$
z(\beta, \bar{\varrho})<1
$$

so that

$$
\left[1+z(\beta, \varrho) \cos \left(\alpha(\bar{\varrho})-\theta_{\varrho}\right)\right] \geqq 0, \text { for all } \varrho \in \mathcal{N}_{\gamma}^{1} .
$$

Moreover, under the same condition,

$$
z(\beta, \bar{\varrho}) \leqq \exp \left[\frac{1}{2}\left(\beta_{1}-\beta / 216\right)\|\varrho\|_{2}^{2}\right] \exp \left[\frac{1}{2}\left(\beta_{1}-\beta / 216\right) L(\varrho)\right],
$$

where $L(\varrho)\left(\leqq\|\varrho\|_{2}^{2}\right)$ is the number of links in the support of $\varrho$. 
2.10. Lower Bound on $\langle W(\mathscr{L})\rangle_{\Lambda}(\beta)$ with Perimeter Decay

It follows from (2.76) that for sufficiently large $\beta$

$$
\prod_{\substack{\varrho \in \mathscr{N}_{\gamma}^{1} \\ \delta \varrho=0}}[1+z(\beta, \bar{\varrho}) \cos (\alpha(\bar{\varrho}))] d \mu_{\Lambda}^{0}(\alpha)
$$

is a positive measure. This permits us to apply Jensen's inequality to derive a lower bound on $\langle W(\mathscr{L})\rangle_{\Lambda}(\beta)$. Let $\langle-\rangle_{\mathscr{N}_{\gamma}^{1}}$ denote the normalized expectation corresponding to (2.78).

We shall make use of the following simple estimate:

$$
\begin{aligned}
1+z \cos (\alpha-\theta) & =(1+z \cos \alpha)\left[1+\frac{z \cos \alpha(\cos \theta-1)+z \sin \alpha \sin \theta}{1+z \cos \alpha}\right] \\
& \geqq(1+z \cos \alpha) e^{E(\alpha, \theta)} e^{O(\alpha, \theta)} e^{-2\left(\frac{z}{1-z}\right)^{2} \theta^{2}},
\end{aligned}
$$

where

$$
E(\alpha, \theta)=(1+z \cos \alpha)^{-1} z \cos \alpha(\cos \theta-1),
$$

and

$$
O(\alpha, \theta)=(1+z \cos \alpha)^{-1} z \sin \alpha \sin \theta .
$$

This inequality follows from Taylor's theorem with remainder, applied to the function $\log (1+x)$, along with elementary estimates on trigonometric functions.

Thus, by Jensen's inequality,

$$
\begin{aligned}
& \int \prod_{\substack{\varrho \in \mathcal{N}^{1} \\
\delta \varrho}}\left[1+z(\beta, \bar{\varrho}) \cos \left(\alpha(\varrho)-\theta_{\varrho}\right)\right] d \mu_{\Lambda}^{0}(\alpha) \\
& \geqq Z_{\mathcal{N}_{\gamma}^{1}} \prod_{\substack{\varrho \in \mathscr{N}^{1} \\
\delta \varrho=0}}\left\{e^{-\left\langle E\left(\alpha(\bar{\varrho}), \theta_{\varrho}\right)\right\rangle_{\mathcal{Y}}} e^{-\left\langle O\left(\alpha(\bar{\varrho}), \theta_{\varrho}\right)\right\rangle_{\mathcal{V}_{\nu}^{1}}} e^{-2(z(\beta, \bar{\varrho}) / 1-z(\beta, \bar{\varrho}))^{2} \theta_{\varrho}^{2}}\right\} .
\end{aligned}
$$

But $\left\langle O\left(\alpha(\bar{\varrho}), \theta_{\varrho}\right)\right\rangle_{\mathcal{N}_{\gamma}^{\frac{1}{\gamma}}}=0$, since $O$ is odd in $\alpha$, while $\langle-\rangle_{\mathcal{N}_{\gamma}^{\frac{1}{\gamma}}}$ is even in $\alpha$, and

$$
\left\langle E\left(\alpha(\varrho), \theta_{\varrho}\right)\right\rangle_{\mathcal{N}_{\nu}^{1}} \leqq 1 / 2 \frac{z(\beta, \bar{\varrho})}{1-z(\beta, \bar{\varrho})} \theta_{\varrho}^{2} .
$$

We now set

$$
\theta_{\varrho}=\left(\varepsilon_{\Lambda}, \mu_{\varrho}\right)_{\Lambda^{*}} \text { and } \gamma(z)=1 / 2 \frac{z}{1-z}+2 \frac{z^{2}}{(1-z)^{2}} .
$$

By combining Corollary 4 with inequality (2.79) we obtain the lower bound

$$
\langle W(\mathscr{L})\rangle_{\Lambda}(\beta) \geqq e^{-(1 / 2 \beta)\left(\varepsilon_{\Lambda}, \varepsilon_{\Lambda}\right)}\left\{\sum_{\gamma} \lambda_{\mathcal{N}_{\gamma}^{1}} \prod_{\substack{\varrho \in \mathcal{N}^{1} \\ \delta \varrho=0}} e^{-\gamma(z(\beta, \bar{\varrho})) \theta_{\varrho}^{2}}\right\},
$$

where $0 \leqq \lambda_{\mathcal{N}_{\gamma}^{\frac{1}{\gamma}}} \equiv d_{\gamma}\left(Z_{\mathcal{N}_{\gamma}^{\frac{1}{\gamma}}} / \Xi_{\Lambda}\right)$, and $Z_{\mathscr{N}_{\gamma}^{\frac{1}{\gamma}}}$ is the total mass of the measure (2.78). By Corollary 4 ,

$$
\sum \lambda_{\mathscr{N}_{\gamma}^{1}}=1
$$


Next, by Lemma 1, Sect. 2.3, and the definition of $\mu_{\varrho}$, see (2.56), Sect. 2.7,

$$
\left|\theta_{\varrho}\right| \equiv\left|\left(\varepsilon_{\Lambda}, \mu_{\varrho}\right)_{\Lambda}\right| \leqq \max _{p \in \Omega_{\varrho}}\left|\varepsilon_{\Lambda}(p)\right| \max _{p}\left|\mu_{\varrho}(p)\right| \operatorname{card}\left(\Omega_{\varrho}\right) .
$$

For each $\varrho \in \mathscr{N}_{\gamma}^{1}$, we now choose a plaquette $p(\varrho)$ containing a link in supp $\varrho$ and such that $p\left(\varrho_{1}\right) \neq p\left(\varrho_{2}\right)$, for any two current densities $\varrho_{1} \neq \varrho_{2}$ in $\mathscr{N}_{\gamma}^{1}$. By the definition of $\Omega_{\varrho}$ (see Lemma 1, Sect. 2.3),

$$
\max _{p \in \Omega_{\varrho}}\left|\varepsilon_{\Lambda}(p)\right| \leqq\left|\varepsilon_{\Lambda}(p(\varrho))\right| \cdot\left[1+\max _{|p-p(\varrho)| \leqq c \cdot L(\varrho)} \frac{\left|\varepsilon_{\Lambda}(p)-\varepsilon_{\Lambda}(p(\varrho))\right|}{\left|\varepsilon_{\Lambda}(p(\varrho))\right|}\right]
$$

for some geometrical constant $c$.

We now recall definition $(2.51)$, Sect. 2.7 , of $\varepsilon_{\Lambda}$. From that definition it follows that

$$
\max _{|p-p(\varrho)| \leqq c \cdot L(\varrho)} \frac{\left|\varepsilon_{\Lambda}(p)-\varepsilon_{\Lambda}(p(\varrho))\right|}{\left|\varepsilon_{\Lambda}(p(\varrho))\right|} \leqq \operatorname{const} L(\varrho)^{3} .
$$

Moreover by Lemma 1, Sect. 2.3,

$$
\max _{p}\left|\mu_{\varrho}(p)\right| \leqq\|\varrho\|_{1} \leqq\|\varrho\|_{2}^{2} .
$$

Finally,

$$
\operatorname{card}\left(\Omega_{\varrho}\right) \leqq \operatorname{const} L(\varrho)^{4}
$$

(an elementary isoperimetric inequality). Let $c(\beta) \equiv 1 / 2\left(\beta_{1}-\beta / 216\right)$. We choose $\beta$ so large that

$$
z(\beta, \bar{\varrho}) \leqq e^{-c(\beta)\|\varrho\|_{2}} e^{-c(\beta) L(\varrho)} \leqq 1-\delta,
$$

for some $\delta>0$, for all $\varrho \in \mathscr{N}_{\gamma}^{1}$ and all $\gamma$ [see (2.77), Sect. 2.9].

We then derive from (2.82)-(2.86) that

$$
\begin{aligned}
\gamma(z(\beta, \bar{\varrho})) \theta_{\varrho}^{2} \leqq & \operatorname{const}\left\{e^{-c(\beta)\|\varrho\|_{2}^{2}}\|\varrho\|_{2}^{4}\right. \\
& \left.\cdot e^{-c(\beta) L(\varrho)} L(\varrho)^{14}\right\}\left|\varepsilon_{\Lambda}(p(\varrho))\right|^{2} \\
\leqq & d(\beta)\left|\varepsilon_{\Lambda}(p(\varrho))\right|^{2},
\end{aligned}
$$

for some finite constant $d(\beta)$.

By (2.80), (2.81), and (2.87),

$$
\begin{aligned}
\langle W(\mathscr{L})\rangle_{\Lambda}(\beta) & \geqq \exp \left[-\{(1 / 2 \beta)+d(\beta)\}\left(\varepsilon_{\Lambda}, \varepsilon_{\Lambda}\right)\right] \\
& =\exp \left[-\left(1 / 2 \beta^{\prime}\right)\left(\varepsilon_{\Lambda}, \varepsilon_{\Lambda}\right)\right] ;
\end{aligned}
$$

with $\beta^{\prime}=1 / 2((1 / 2 \beta)+d(\beta))^{-1}$.

The right side of (2.88) is a Gaussian expectation value of $D_{\partial \Sigma}(\alpha)$ [see (2.54), Sect. 2.7]. Recalling the definition (2.51), Sect. 2.7, of $\varepsilon_{\Lambda}$, we observe that

$$
\left(\varepsilon_{\Lambda}, \varepsilon_{\Lambda}\right) \leqq \operatorname{const}(L+T),
$$

as $\Lambda \nearrow \mathbb{Z}^{4}$.

This completes our proof of perimeter decay of $\langle W(\mathscr{L})\rangle(\beta)$, for sufficiently large $\beta$. 
This result can be extended to the compact $U(1)$ model on $\mathbb{Z}^{4}$ with Wilson's action by combining the present techniques with an adaptation of Appendix B, Lemma 4.3 and of the methods in Sect. 6 of [15] to the $U(1)$ gauge theory. Since, due to the analytical subtleties of modified Bessel functions, the details are rather lengthy but fairly uninteresting we do not wish to present them here. (The reader familiar with [15] will have no problems to supply them; see also Sect. 3.)

\subsection{Masslessness of the Photon for Large $\beta$}

We finally prove a result which we believe is new and somewhat important.

The lattice approximation of the electromagnetic field strength is given by

$$
\Phi_{p} \equiv \begin{cases}-i\left(\frac{\partial}{\partial \theta} \varphi_{\beta}\right)\left(d \theta_{p}\right) \varphi_{\beta}\left(d \theta_{p}\right)^{-1}, & \text { for the } U(1) \text {-model with Villain action } \\ i \beta \sin \left(d \theta_{p}\right), & \text { for Wilson's } U(1) \text {-model }\end{cases}
$$

We propose to show that, for large $\beta$, the two-point (more precisely: twoplaquette) correlation of $\Phi_{p}$ cannot have summable ("integrable") fall-off. This proves that the large $\beta$ phase of the compact $U(1)$ model is massless, i.e. the photon is massless, for sufficiently large $\beta$.

As in previous sections, we only present the proof for the Villain approximation to the compact $U(1)$ model. Most of our arguments extend, however, to a general class of actions, and we believe that the result is a general feature of the $U(1)$ model in the weak coupling regime.

The observable corresponding to $\Phi_{p}$, after a duality transformation, is $(d \alpha)_{p^{*}}$ It is therefore enough to estimate the behaviour of the two-point functions $\left\langle|\alpha(\mu)|^{2}\right\rangle(\beta)$, where $\mu$ is an arbitrary 1 -form satisfying $\delta \mu=0$. We propose to prove that

$$
\beta^{\prime \prime}\left(\mu,(-\Delta)^{-1} \mu\right) \leqq\left\langle|\alpha(\mu)|^{2}\right\rangle(\beta) \leqq \beta\left(\mu,(-\Delta)^{-1} \mu\right),
$$

for some function $\beta^{\prime \prime}(\beta)<\beta$ which diverges to $+\infty$, as $\beta \rightarrow \infty$.

Let $(d \alpha)_{\mu \nu}$ denote the $\mu \nu$-component of the curl of $\alpha$ (the field strength), and let $\widehat{(d \alpha}_{\mu \nu}$ denote its Fourier transform. By Fourier transformation, (2.90) provides a lower and an upper bound on

$$
\left\langle\left|\widehat{(d \alpha)_{\mu v}}(k)\right|^{2}\right\rangle(\beta)
$$

in terms of an expectation value of $\mid\left(\left.\widehat{d \alpha}_{\mu \nu}(k)\right|^{2}\right.$ in the Gaussian measure $d \mu^{0}(\alpha)$ with charge $g^{2}=\beta^{-1},\left(\beta^{\prime \prime}\right)^{-1}$, respectively. These Gaussian expectations are well known to be discontinuous at $k=0$ :

$$
\begin{aligned}
& \lim _{\mid \hat{k \mid \rightarrow 0}}\left\langle\left|\widehat{(d \alpha)}_{\mu \nu}(k)\right|^{2}\right\rangle^{0}(\beta)=0 \\
& \varlimsup_{|k| \rightarrow 0}\left\langle\left|\widehat{d \alpha)_{\mu \nu}}(k)\right|^{2}\right\rangle^{0}\left(\beta^{\prime \prime}\right)>0 .
\end{aligned}
$$

Thus, $\left\langle\left|\widehat{(d \alpha)}_{\mu v}(k)\right|^{2}\right\rangle(\beta)$ is discontinuous at $k=0$. As a consequence,

$$
\left\langle(d \alpha)_{\mu \nu}(p)(d \alpha)_{\mu \nu}\left(p^{\prime}\right)\right\rangle(\beta)
$$


cannot have summable fall-off, as $\operatorname{dist}\left(p, p^{\prime}\right) \rightarrow \infty$. [Here $p$ and $p^{\prime}$ are two arbitrary plaquettes parallel to the $\mu \nu$-lattice plane, and $\left.(d \alpha)_{\mu \nu}(p) \equiv(d \alpha)_{p^{*}}\right]$

This proves our contention. (See also [21] for more details concerning a similar argument for dipole gases.)

Next, we note that, by polarization, it suffices to prove (2.90) for real-valued 1 -forms, $\mu$, with $\delta \mu=0$, i.e.

$$
\beta^{\prime \prime}\left(\mu,(-\Delta)^{-1} \mu\right) \leqq\left\langle\alpha(\mu)^{2}\right\rangle(\beta) \leqq \beta\left(\mu,(-\Delta)^{-1} \mu\right),
$$

with $\beta^{\prime \prime}$ as in $(2.90), \mu$ real.

A stronger version of $(2.92)$ is

$$
\begin{aligned}
& \exp \left[\frac{\varepsilon^{2} \beta^{\prime \prime}}{2}\left(\mu,(-\Delta)^{-1} \mu\right)\right] \\
& \leqq\left\langle e^{\varepsilon \alpha(\mu)}\right\rangle(\beta) \\
& \leqq \exp \left[\frac{\varepsilon^{2} \beta}{2}\left(\mu,(-\Delta)^{-1} \mu\right)\right],
\end{aligned}
$$

for arbitrary real $\varepsilon$ and real $\mu$, with $\delta \mu=0$. By expanding (2.93) in powers of $\varepsilon$, subtracting 1 , dividing by $\varepsilon^{2}$ and taking the limit $\varepsilon=0$, (2.92) follows.

Finally, it is clearly enough to prove (2.93) in an arbitrary, finite region $\Lambda$, replacing $(-\Delta)^{-1}$ by $V_{\Lambda}$, and $\langle-\rangle(\beta)$ by $\langle-\rangle_{\Lambda}(\beta)$.

\subsection{Proof of (2.93) in Finite Volume}

We fix a real 1 -form $\mu$, with $\delta \mu=0$ and such that $\operatorname{supp} \mu$ is in the interior of $\Lambda^{*}$. We then define a 1 -form, $\tau$, by

$$
\tau=\varepsilon \beta V_{\Lambda} \mu,
$$

where $V_{A}$ is the Green's function of $\Pi_{A^{*}} \delta d$. Next, by (2.47), Sect. 2.6

$$
\Xi_{\Lambda} \cdot d \mu_{\Lambda}(\alpha)=\sum_{\gamma} d_{\gamma} \prod_{\substack{\varrho \in \mathcal{N}_{\gamma}^{1} \\ \delta \varrho=0}}[1+K(\varrho) \cos (\alpha(\varrho))] d \mu_{\Lambda}^{0}(\alpha) .
$$

We now change variables,

$$
\alpha \rightarrow \alpha+\tau,
$$

with $\tau$ given by (2.94).

By (2.52) and (2.55), Sect. 2.7,

$$
\begin{aligned}
d \mu_{\Lambda}(\alpha) \rightarrow d \mu_{\Lambda}(\alpha+\tau)= & e^{-(1 / \beta)(d \alpha, d \tau)_{\Lambda^{*}}} e^{-(1 / 2 \beta)(d \tau, d \tau)_{\Lambda^{*}}} . \\
& \cdot \Xi_{\Lambda}^{-1}\left\{\sum_{\gamma} d_{\gamma} \prod_{\substack{\varrho \in \mathscr{N}_{\gamma}^{1} \\
\delta \varrho=0}}\left[1+K(\varrho) \cos \left(\alpha(\varrho)+d \tau\left(\mu_{\varrho}\right)\right)\right] d \mu_{\Lambda}^{0}(\alpha)\right\},(2.95)
\end{aligned}
$$

with $\delta \mu_{\varrho}=\varrho$, for all $\varrho$.

Moreover,

$$
e^{\varepsilon \alpha(\mu)} \rightarrow e^{\varepsilon \alpha(\mu)} e^{\varepsilon \tau(\mu)}
$$


We now observe that

and

$$
\begin{aligned}
-(1 / \beta)(d \alpha, d \tau)_{\Lambda^{*}} & =-\varepsilon \alpha(\mu), \\
-(1 / 2 \beta)(d \tau, d \tau)_{\Lambda^{*}} & =-\frac{\varepsilon}{2} \tau(\mu),
\end{aligned}
$$

$$
\tau(\mu)=\varepsilon \beta\left(\mu, V_{\Lambda} \mu\right)
$$

Therefore

$$
\left\langle e^{\varepsilon \alpha(\mu)}\right\rangle_{\Lambda}(\beta)=e^{\left(\varepsilon^{2} \beta / 2\right)\left(\mu, V_{\Lambda} \mu\right)} \Xi_{\Lambda}^{-1}\left\{\sum_{\gamma} d_{\gamma} \mathscr{I}_{\gamma}(\tau)\right\}
$$

where

$$
\mathscr{I}_{\gamma}(\tau)=\int \prod_{\substack{\varrho \in \mathcal{N}^{\frac{1}{\gamma}} \\ \delta \varrho=0}}\left[1+K(\varrho) \cos \left(\alpha(\varrho)+d \tau\left(\mu_{\varrho}\right)\right)\right] d \mu_{\Lambda}^{0}(\alpha) .
$$

Since $d \mu_{\Lambda}^{0}(\alpha)$ and $\cos (\alpha(\varrho))$ are of positive type in $\alpha$, and $K(\varrho)>0$, for all $\varrho$, we immediately conclude that

$$
\mathscr{I}_{\gamma}(\tau) \leqq \mathscr{I}_{\gamma}(\tau \equiv 0){ }^{3}
$$

Since

$$
\sum_{\gamma} d_{\gamma} \mathscr{I}_{\gamma}(\tau \equiv 0) \equiv \Xi_{\Lambda}
$$

the upper bound in (2.93) follows from (2.97) and (2.99), by letting $\Lambda \uparrow \mathbb{Z}^{4}$.

Finally, we establish a lower bound on $\mathscr{I}_{\gamma}(\tau)$. This is achieved by using the results in Sects. 2.8-2.10, with

$$
\theta_{\varrho}=d \tau\left(\mu_{\varrho}\right), \quad\left(d \mu_{\varrho}=\varrho\right) .
$$

By (2.79) and (2.80), Sect. 2.10,

$$
\mathscr{I}_{\gamma}(\tau) \geqq \sum_{\gamma} \lambda_{\mathcal{N}_{\gamma}^{1}} \prod_{\substack{\varrho \in \mathcal{N}^{\mathcal{\beta}} \\ \delta \varrho=0}} e^{-\gamma(z(\beta, \bar{\varrho})) \theta^{2}},
$$

for sufficiently large $\beta$, where

$$
\begin{aligned}
\lambda_{\mathcal{N}_{\gamma}^{1}} & \equiv \Xi_{\Lambda}^{-1} d_{\gamma} Z_{\mathscr{N}_{\gamma}^{1}} \\
Z_{\mathcal{N}_{\gamma}^{1}} & =\int \prod_{\substack{\varrho \in \mathscr{N}^{1} \\
\delta \varrho}}[1+z(\beta, \bar{\varrho}) \cos \alpha(\bar{\varrho})] d \mu_{\Lambda}^{0}(\alpha),
\end{aligned}
$$

and

$$
\gamma(z) \leqq 4 z, \text { for } z \leqq 1 / 2
$$

see (2.78)-(2.80), Sect. 2.10 .

3 This part of the argument does not obviously extend to Wilson's form of the $U(1)$ model and has to be replaced by a more complicated, direct one 
By (2.77)

$$
\begin{aligned}
z(\beta, \varrho) \leqq & \exp \left[\frac{1}{2}\left(\beta_{1}-\beta / 216\right)\|\varrho\|_{2}^{2}\right] \\
& \cdot \exp \left[\frac{1}{2}\left(\beta_{1}-\beta / 216\right) L(\varrho)\right]
\end{aligned}
$$

where $\beta_{1}$ is a finite constant, and $L(\varrho)$ is the number of links in supp $\varrho$, provided

$$
\beta>216 \beta_{1} \text {. }
$$

Next, using Lemma 1, Sect. 2.3, one finds

$$
\begin{aligned}
\left|\theta_{\varrho}\right| \equiv\left|d \tau\left(\mu_{\varrho}\right)\right| & \leqq \max _{p \in \Omega_{\varrho}}\left|(d \tau)_{p}\right| \cdot\|\varrho\|_{1} \operatorname{card}\left(\Omega_{\varrho}\right) \\
& \leqq \max _{p \in \Omega_{\varrho}}\left|(d \tau)_{p}\right| \cdot\|\varrho\|_{2}^{2} \cdot \operatorname{const} L(\varrho)^{4},
\end{aligned}
$$

see also (2.82).

We now fix a plaquette, $p_{0}$, and a positive integer $L$. We must estimate the cardinality of the set, $\mathscr{N}_{\tau}\left(p_{0}, L\right)$, of current distributions defined by

$$
\begin{aligned}
\mathscr{N}_{\tau}\left(p_{0}, L\right) & \equiv\left\{\varrho \in \mathscr{N}_{\gamma}^{1}: \max _{p \in \Omega_{\varrho}}\left|(d \tau)_{p}\right|=\left|(d \tau)_{p_{0}}\right|, L(\varrho)=L\right\} \\
& \leqq\left\{\varrho: p_{0} \subset \Omega_{\varrho}, L(\varrho)=L\right\} .
\end{aligned}
$$

Clearly, the length of the edges of $\Omega$, for some $\varrho$ satisfying $L(\varrho)=L$, is bounded by $L$. Thus the support of every $\varrho$ with the properties

$$
L(\varrho)=L \text { and } \Omega_{\varrho} \supset p_{0}
$$

is contained in a cube with edges of length at most $2 L$.

Given a cube, $\Omega$, with edges of length $2 L$, the maximal number of current distributions $\left\{\varrho_{j}\right\} \subset \mathscr{N}_{\gamma}^{1}$ with disjoint supports, all contained in $\Omega$, and $L\left(\varrho_{j}\right)=L$, for all $j$, is bounded by

$$
4(2 L)^{4} / L=64 L^{3}
$$

Thus, for $\beta$ so large that $\tilde{\beta} \equiv 1 / 2\left(\beta_{1}-\beta / 216\right)>0$,

$$
\begin{aligned}
\sum_{\varrho \in \mathcal{N}_{\tau}\left(p_{0}, L\right)} \gamma(z(\beta, \varrho)) \theta_{\varrho}^{2} & \leqq\left|(d \tau)_{p_{0}}\right|^{2} \sum_{\varrho \in \mathcal{N}_{\tau}\left(p_{0}, L\right)}\|\varrho\|_{2}^{2} \operatorname{const} L^{8} \gamma(z(\beta, \varrho)) \\
& \leqq\left|(d \tau)_{p_{0}}\right|^{2} \operatorname{const} L^{11} e^{-\tilde{\beta} L} \max \left(\|\varrho\|_{2}^{2} e^{-\tilde{\beta}\|\varrho\|_{2}^{2}}\right) \\
& \leqq \operatorname{const} \tilde{\beta}^{-1}\left|(d \tau)_{p_{0}}\right|^{2} L^{11} e^{-\tilde{\beta} L} .
\end{aligned}
$$

Hence

$$
\begin{aligned}
& \sum_{L=4}^{\infty} \sum_{\varrho \in \mathcal{N}_{\tau}\left(p_{0}, L\right)} \gamma(z(\beta, \bar{\varrho})) \theta_{\varrho}^{2} \\
& \quad \leqq \operatorname{const} \tilde{\beta}^{-1}\left|(d \tau)_{p_{0}}\right|^{2}\left\{\sum_{L=4}^{\infty} L^{11} e^{-\bar{\beta} L} \leqq c(\beta)\left|(d \tau)_{p_{0}}\right|^{2}\right\}
\end{aligned}
$$

for some function $c(\beta)$ which tends to 0 , as $\beta \rightarrow \infty$, exponentially fast. [We have used that $\mathscr{N}_{\tau}\left(p_{0}, L\right)=\emptyset$, for $L<4$.] 
If we now insert (2.107) into (2.101) we find

$$
\begin{aligned}
\mathscr{I}_{\gamma}(\tau) & \geqq e^{-c(\beta)\|d \tau\|_{2}^{2}} \sum_{\gamma} \lambda_{\mathcal{N}_{\gamma}^{1}} \\
& =e^{-c(\beta)\|d \tau\|_{2}^{2}} .
\end{aligned}
$$

By (2.97),

$$
\begin{aligned}
\left\langle e^{\varepsilon \alpha(\mu)}\right\rangle_{\Lambda}(\beta) & \geqq e^{\left(\varepsilon^{2} \beta / 2\right)\left(\mu, V_{\Lambda} \mu\right)} e^{-c(\beta)\|d \tau\|_{2}^{2}} \\
& =e^{\varepsilon^{2}(\beta / 2)[1-2 c(\beta) / \beta]\left(\mu, V_{\Lambda} \mu\right)},
\end{aligned}
$$

where we have used (2.96).

This completes our proof of the basic lower bound (2.93) in finite volume and thus of the masslessness of the photon for large $\beta$.

Remarks. 1) Using correlation inequalities [17, 24] one derives from the results in this section the existence of massless, deconfining phases in all $D$-dimensional $U(1)$ gauge theories with $D \geqq 4$. Alternatively, a direct proof can be given by using a duality transformation and a straightforward modification of the techniques developed in this section (see also [15] and Sect. 4).

2) It appears that the techniques of this section along with connections between the four-dimensional, dual $U(1)$ theory and bond percolation are useful to study the scaling limits for large $\beta$ (ordinary, free QED) and for $\beta \uparrow \beta_{\text {crit }}$ (massive, confining QED). Our ideas and some results on bond percolation suggest that the latter theory might be a non-trivial, confining version of QED.

\section{QED Phases in the Four-Dimensional $\mathbb{Z}_{N}$ Lattice Gauge Theories, for Large $N$}

\subsection{Preliminary Remarks}

In this section we prove inequalities (1.13) and (1.15), i.e.

$$
\left.\begin{array}{r}
\left\langle W\left(\mathscr{L}_{L \times T}\right)\right\rangle^{(N)}(\beta) \geqq \exp [-d(L+T)], \\
\left\langle D_{\mathscr{L}_{L \times T}}\right\rangle^{(N)}(\beta) \geqq \exp [-\delta(L+T)],
\end{array}\right\}
$$

for the four-dimensional $\mathbb{Z}_{N}$ models, for all

$$
\beta>\beta_{\text {crit }}(U(1))
$$

[the critical value of $\beta$ for the $U(1)$ model], and all

$$
N>N(\beta)
$$

where $N(\beta)$ is an integer-valued function of $\beta$ which diverges to $+\infty$, as $\beta \rightarrow \infty$. Here \langle\rangle$^{(N)}(\beta)$ is the infinite volume state of the $\mathbb{Z}_{N}$ model at "temperature" $\beta^{-1}=g^{2}$. It follows that for

$$
N>N_{c}, \quad \text { with } \quad N_{c} \leqq N\left(\beta_{\text {crit }}(U(1))<\infty\right.
$$

there exist $\underline{\beta}_{c}(N)$ and $\bar{\beta}_{c}(N)$, with

$$
\underline{\beta}_{c}(N)<\bar{\beta}_{c}(N)<\infty \text {, and } \underline{\beta}_{c}(N) \leqq \beta_{\text {crit }}(U(1)),
$$


such that for

$$
\underline{\beta}_{c}(N)<\beta<\bar{\beta}_{c}(N)
$$

both inequalities in (3.1) hold.

A standard high temperature expansion shows that $\langle W(\mathscr{L})\rangle^{(N)}(\beta)$ has area decay, for sufficiently small $\beta$ (depending on $N$ ), and a low temperature expansion (or a high temperature expansion applied to the dual model) can be used to prove that $\left\langle D_{\mathscr{L}}\right\rangle^{(N)}(\beta)$ has area decay when $\beta$ is sufficiently large (depending on $N$ ).

Thus, for $N>N_{c}$, the $\mathbb{Z}_{N}$ models have a "quark" confining high temperature phase and a "magnetic monopole" confining low temperature phase, separated by an open interval, $\left(\beta_{c}(N), \bar{\beta}_{c}(N)\right)$, of QED phases. It is believed that $N_{c}=5$.

For the Villain approximation of the $\mathbb{Z}_{N}$ models this result follows from [13] by using self-duality and correlation inequalities, as shown in [16].

We reconsider the $\mathbb{Z}_{N}$ models for the following reasons:

1) Our method will not rely on self-duality. This permits us to analyze a large class of actions, including Wilson's action, and to exhibit intermediate QED phases in $D$-dimensional $\mathbb{Z}_{N}$ lattice gauge theories for arbitrary $D \geqq 4$.

2) Our methods involve a renormalization transformation which maps some class of $\mathbb{Z}_{N}$ expectations in the intermediate QED phase onto expectations in a model with local U(1) gauge invariance. (This is the phenomenon described in Sect. 1.)

\subsection{Interpolation Between the $U(1)$ and $\mathbb{Z}_{N}$ Models}

We consider a family of models interpolating between the $U(1)$ and a $\mathbb{Z}_{N}$ model. Let $d \mu_{\beta}(\theta)$ denote the infinite volume limit of the measures

$$
\begin{gathered}
d \mu_{\beta}\left(\theta_{\Lambda}\right)=Z_{\beta, \Lambda}^{-1} \prod_{p \subset \Lambda} e^{\beta \cos \left(d \theta_{p}\right)} \prod_{x y \subset \Lambda} d \theta_{x y}, \\
d \theta_{p}=\sum_{x y \subset \partial p p} \theta_{x y}, \quad \Lambda \subset \mathbb{Z}^{4},
\end{gathered}
$$

which correspond to the four-dimensional $U(1)$-model with Wilson action in a finite region $\Lambda$. Instead, we could define $d \mu_{\beta}\left(\theta_{\Lambda}\right)$ to be the finite volume functional measure of the Villain model by replacing $\exp \beta \cos \left(d \theta_{p}\right)$ by $\sum_{n=-\infty}^{\infty} \exp \left[-\frac{\beta}{2}\left(d \theta_{p}+2 \pi n\right)^{2}\right]$. In both cases the limit $\Lambda \uparrow \mathbb{Z}^{4}$ exists, thanks to Ginibre's inequalities [17].

We now define

$$
d \mu_{\beta}^{h}\left(\theta_{\Lambda}\right)=\left(Z_{\beta, \Lambda}^{h}\right)^{-1} \prod_{x y \subset \Lambda} \xi(h) e^{h \cos \left(N \theta_{x y}\right)} d \mu_{\beta}(\theta),
$$

where

and

$$
\left.\begin{array}{rl}
\xi(h) & =\left(\frac{1}{2 \pi} \int_{0}^{2 \pi} e^{h \cos (N \theta)} d \theta\right)^{-1}, \\
Z_{\beta, \Lambda}^{h} & =\int \prod_{x y \subset \Lambda} \xi(h) e^{h \cos \left(N \theta_{x y}\right)} d \mu_{\beta}(\theta) .
\end{array}\right\}
$$

Clearly, $d \mu_{\beta}^{h}\left(\theta_{\Lambda}\right)$ approaches the Euclidean functional measure of the $\mathbb{Z}_{N}$ model in a finite region $\Lambda$ with free boundary conditions (b.c.), as $h \rightarrow \infty$. [Actually b.c. turn out 
to be quite irrelevant in our analysis : We could replace $d \mu_{\beta}(\theta)$ by $d \mu_{\beta}\left(\theta_{A}\right)$ in (3.7) and (3.8) which would merely slightly complicate notations in subsequent formulas.]

Let $\langle-\rangle(\beta)$ denote the $U(1)$ expectation, and $\langle-\rangle_{A}(\beta, h)$ the one determined by the measure (3.7). By Ginibre's inequality [17], $\left\langle W\left(\mathscr{L}_{L \times T}\right)\right\rangle_{\Lambda}(\beta, h)$ is monotone increasing in $\Lambda$ and in $h$, so that

$$
\left\langle W\left(\mathscr{L}_{L \times T}\right)\right\rangle_{\Lambda}(\beta, h) \geqq\left\langle W\left(\mathscr{L}_{L \times T}\right)\right\rangle(\beta),
$$

for arbitrary $\Lambda \subseteq \mathbb{Z}^{4}, h \geqq 0$, and

$$
\begin{aligned}
\left\langle W\left(\mathscr{L}_{L \times T}\right)\right\rangle^{(N)}(\beta) & =\lim _{\Lambda^{\uparrow} \mathbb{Z}^{4}} \lim _{h \uparrow \infty}\left\langle W\left(\mathscr{L}_{L \times T}\right)\right\rangle_{\Lambda}(\beta, h) \\
& =\lim _{h \uparrow \infty}\left\langle W\left(\mathscr{L}_{L \times T}\right)\right\rangle(\beta, h) .
\end{aligned}
$$

Thus, for $\beta>\beta_{\text {crit }}(U(1))$,

$$
\begin{aligned}
\left\langle W\left(\mathscr{L}_{L \times T}\right)\right\rangle^{(N)}(\beta) & \geqq\left\langle W\left(\mathscr{L}_{L \times T}\right)\right\rangle(\beta, h) \\
& \geqq\left\langle W\left(\mathscr{L}_{L \times T}\right)\right\rangle(\beta) \\
& \geqq \exp [-d(L+T)]
\end{aligned}
$$

which proves the first inequality in (3.1).

\subsection{Disorder Operator}

We now turn to the analysis of the expectation value of the disorder operator and propose to establish perimeter decay for sufficiently small $\beta$.

We closely follow the scheme developed in Sects. 2.4 through 2.10. The first step consists in using the Fourier expansion

$$
\xi(h) \exp \{h \cos (N \theta)\}=1+\sum_{q=1}^{\infty} \lambda(q) \cos (q N \theta),
$$

where

$$
\lambda(q)=\frac{\xi(h)}{\pi} \int_{0}^{2 \pi} \exp h \cos (N \theta) \cos (q N \theta) d \theta
$$

Clearly

$$
0<\lambda(q)<2 \text {, and } \lambda(q) \rightarrow 2, \quad \text { as } \quad h \rightarrow \infty .
$$

Let $\{\zeta(q)\}$ be a sequence of positive numbers with the property that

e.g.

$$
\begin{gathered}
\sum_{q=1}^{\infty} \zeta(q)^{-1}=1, \\
\zeta(q)=c_{\varepsilon} e^{\varepsilon q}, \quad\left(c_{\varepsilon}<\varepsilon^{-1}\right),
\end{gathered}
$$

for some $\varepsilon>0$ chosen later.

Then

$$
1+\sum_{q=1}^{\infty} \lambda(q) \cos (q N \theta)=\sum_{q=1}^{\infty} \zeta(q)^{-1}\left(1+z_{q N} \cos (q N \theta)\right)
$$


with

$$
0<z_{q N}=\zeta(q) \lambda(q)<2 \varepsilon^{-1} e^{\varepsilon q} .
$$

With (3.7) this yields the following expression for the functional measure of the $\mathbb{Z}_{N}$ model in finite volume

$$
d \mu_{\beta}^{h}\left(\theta_{\Lambda}\right)=\left(Z_{\beta, \Lambda}^{h}\right)^{-1} I\left(\theta_{\Lambda}\right) d \mu_{\beta}(\theta),
$$

where

$$
I\left(\theta_{\Lambda}\right)=\sum_{q_{\Lambda}} c_{q_{\Lambda}} \prod_{x y \subset \Lambda}\left(1+z_{N q_{x y}} \cos \left(q_{x y} N \theta_{x y}\right)\right)
$$

and

$$
q_{\Lambda}=\left\{q_{x y}\right\}_{x y \subset \Lambda}, \quad c_{q_{\Lambda}}=\prod_{x y \subset A} \zeta\left(q_{x y}\right)^{-1} .
$$

We now redefine a current distribution, $\varrho$, to be a function on the set, $\mathscr{B}$, of directed bonds in $\Lambda$ with values in $N \mathbb{Z}$, of finite support. A 1 -ensemble, $\mathscr{E}^{1}$, is a family of current distributions, $\varrho$, with the properties

$$
\left.\begin{array}{l}
\operatorname{supp} \varrho \subseteq \Lambda, \quad \text { for all } \varrho \in \mathscr{E}^{1}, \\
\operatorname{dist}\left(\varrho, \varrho^{\prime}\right) \geqq \sqrt{2}
\end{array}\right\}
$$

for all $\varrho$ and $\varrho^{\prime}$ in $\mathscr{E}^{1}$ with $\varrho \neq \varrho^{\prime}$ (see Sect. 2.6). Repeating the combinatorial expansion of Sect. 2.6, see Lemma 2 and (2.46), we obtain

$$
I\left(\theta_{\Lambda}\right)=\sum_{\gamma} d_{\gamma} \prod_{\varrho \in \mathscr{N}_{\gamma}^{1}}(1+K(\varrho) \cos \theta(\varrho)),
$$

where $\theta(\varrho) \equiv \sum_{x y} \theta_{x y} \varrho_{x y}, \gamma$ ranges over a finite index set, each $\mathscr{N}_{\gamma}^{1}$ is a 1 -ensemble, and

ii)

$$
\left.\begin{array}{l}
d_{\gamma}>0, \text { for all } \gamma, \\
0<K(\varrho) \leqq 3^{N_{1}(\operatorname{supp} \varrho)} \prod_{x y \subset \operatorname{supp} \varrho} z_{\left|\varrho_{x y}\right|}
\end{array}\right\}
$$

[We recall that $N_{1}(\operatorname{supp} \varrho)$ is the number of bonds within distance $\leqq 1$ of supp $\varrho$.]

Since the measure $d \mu_{\beta}(\theta)$ is invariant under $U(1)$ gauge transformations, we can impose the condition

$$
\delta \varrho=0
$$

as long as we only want to compute expectations of gauge-invariant observables in the measure $d \mu_{\beta}^{h}\left(\theta_{A}\right)$.

Next, we discuss the expectation value of the disorder operator $D_{\mathscr{L}_{L \times T}}$. We choose the definition of $D_{\mathscr{L}_{L \times T}}$, for $0 \leqq h \leqq \infty$, such that for $h=0$ (U(1) model) and $h=\infty\left(\mathbb{Z}_{N}\right.$ model $)$ it agrees with the one proposed in (1.14). Thus

$$
\begin{aligned}
\left\langle D_{\mathscr{L}_{L \times T}}^{\xi}\right\rangle_{\Lambda}(\beta, h)= & \left(Z_{\beta, \Lambda}^{h}\right)^{-1} \int \prod_{x y \subset \Lambda} \xi(h) e^{h \cos \left(N \theta_{x y}\right)} \\
& \cdot \prod_{p} \exp \beta\left[\cos \left(d \theta_{p}+\varphi_{p}\right)-\cos \left(d \theta_{p}\right)\right] d \mu_{\beta}(\theta),
\end{aligned}
$$


where

$$
\varphi_{p} \equiv \begin{cases}2 \pi \xi / N, & \text { for } p^{*} \in \Sigma, \\ 0, & \text { otherwise }\end{cases}
$$

$\xi=1,2, \ldots, N-1$, and $\Sigma$ is the rectangular array of plaquettes in the $0-1$ plane bounded by $\mathscr{L}_{L \times T}$.

By (3.15), (3.18), and (3.20),

$$
\begin{aligned}
\left\langle D_{\mathscr{L}_{L \times T}}^{\xi}\right\rangle_{\Lambda}(\beta, h)= & \left(Z_{\beta, \Lambda}^{h}\right)^{-1}\left\{\sum_{\gamma} d_{\gamma} \int \prod_{\substack{\varrho \in \mathscr{N}^{1} \\
\delta \varrho=0}}[1+K(\varrho) \cos \theta(\varrho)]\right\} \\
& \cdot \prod_{p} \exp \beta\left[\cos \left(d \theta_{p}+\varphi_{p}\right)-\cos \left(d \theta_{p}\right)\right] d \mu_{\beta}(\theta) .
\end{aligned}
$$

In each term on the right side of (3.23) we make a real change of variables

$$
\theta_{x y} \rightarrow \theta_{x y}+\tau_{x y},
$$

where $\tau$ is the 1 -form determined by

$$
\tau=\delta \Delta^{-1} \varphi,
$$

with $\varphi$ given by (3.22). (We are repeating here the change of variables already used in Sect. 2.7.) Now, notice that

By definition of $\varphi$,

$$
\begin{aligned}
(d \tau)_{p} & =\left(d \delta \Delta^{-1} \varphi\right)_{p} \\
& =-\varphi_{p}-\left(\delta d \Delta^{-1} \varphi\right)_{p} \\
& \equiv-\varphi_{p}+\varepsilon_{p} .
\end{aligned}
$$

$$
*(d \varphi)_{x y}= \begin{cases}2 \pi \xi / N, & \text { for } x y \in \mathscr{L}_{L \times T}, \\ 0, & \text { otherwise }\end{cases}
$$

Hence

$$
\varepsilon_{p} \sim d^{-3},
$$

where $d$ is the distance between $p$ and $\mathscr{L}_{L \times T}$.

Inserting (3.24)-(3.26) into the right side of (3.23) we find, using the periodicity of the cosine and Lemma 1, Sect. 2.3,

$$
\left\langle D_{\mathscr{L}_{L \times T}}\right\rangle_{\Lambda}(\beta, h)=\left(Z_{\beta, \Lambda}^{h}\right)^{-1} \sum_{\gamma} d_{\gamma} \mathscr{I}_{\gamma}(\varepsilon)
$$

where

$$
\mathscr{I}_{\gamma}(\varepsilon)=\int \prod_{\substack{\varrho \in \mathscr{N}_{\gamma}^{1} \\ \delta \varrho=0}}\left[1+K(\varrho) \cos \left(\theta(\varrho)+\varepsilon\left(\mu_{\varrho}\right)\right)\right] \mathscr{R}(d \theta+\varepsilon) d \mu_{\beta}(\theta),
$$

and

$$
\begin{aligned}
\mathscr{R}(d \theta+\varepsilon) & \equiv \prod_{p} \exp \beta\left[\cos \left(d \theta_{p}+d \tau_{p}+\varphi_{p}\right)-\cos \left(d \theta_{p}\right)\right] \\
& =\prod_{p} \exp \beta\left[\cos \left(d \theta_{p}+\varepsilon_{p}\right)-\cos \left(d \theta_{p}\right)\right] .
\end{aligned}
$$


[We have used (3.26), the fact that $\mu_{\varrho}$ takes values in $N \mathbb{Z}$ and the periodicity of the cosine to get rid of $\varphi$.]

\subsection{The Renormalization Transformation}

Next, we must perform the renormalization transformation. It is a straightforward variant of the one described in Sect. 2.8. (We draw on some ideas from Sect. 4 of [15].)

Given any current distribution $\varrho$ in a 1 -ensemble, $\mathcal{N}_{\gamma}^{1}$, we choose a set of links $\mathscr{B}_{\varrho}$ contained in supp $\varrho$, with the property that two different links in $\mathscr{B}_{\varrho}$ do not belong to a common plaquette and such that

$$
\sum_{x y \in \mathscr{B}_{\varrho}}\left|\varrho_{x y}\right| \geqq(1 / 18)\|\varrho\|_{1},
$$

see (2.62), Sect. 2.8. Since

$$
\operatorname{dist}\left(\varrho_{1}, \varrho_{2}\right) \geqq \sqrt{2},
$$

for any two distributions $\varrho_{1}, \varrho_{2}$ in $\mathscr{N}_{\gamma}^{1}, \varrho_{1} \neq \varrho_{2}$, the choice of $\mathscr{B}_{\varrho}$ only depends on $\varrho$ but is independent of $\mathscr{N}_{\gamma}^{1} \sim\{\varrho\}$, and there is no plaquette containing a link of $\mathscr{B}_{\varrho}$ and a link of $\mathscr{B}_{\varrho^{\prime}}$, for any $\varrho^{\prime} \in \mathscr{N}_{\gamma}^{1}$.

Our renormalization transformation consists of integration out all variables

$$
\left\{\theta_{x y}: x y \in \mathscr{B}_{\varrho}, \varrho \in \mathscr{N}_{\gamma}^{1}\right\} .
$$

As in the proof of Lemma 3, Sect. 2.8, one sees that this can be reduced to evaluating the integrals

$$
S\left(\varrho_{x y}\right) \equiv \int e^{i \varrho_{x y} \theta_{x y}} \prod_{p: \partial p \supset x y} e^{\beta \cos \left(d \theta_{p}+\varepsilon_{p}\right)} d \theta_{x y},
$$

$x y \in \mathscr{B}_{\varrho}, \varrho \in \mathscr{N}_{\gamma}^{1}$. This is achieved by performing a complex translation,

$$
\theta_{x y} \rightarrow \theta_{x y}+i \alpha
$$

(see also Lemma 4.3 of [15]). Under this change of variables

$$
\begin{aligned}
S\left(\varrho_{x y}\right)= & e^{-\alpha \varrho_{x y}} e^{6 \beta(\cosh \alpha-1)} \int e^{i \varrho_{x y} \theta_{x y}} \\
& \cdot \prod_{p: \partial p \supset x y} i_{\beta}\left(\alpha ; d \theta_{p}+\varepsilon_{p}\right) e^{\beta \cos \left(d \theta_{p}+\varepsilon_{p}\right)} d \theta_{x y},
\end{aligned}
$$

where

$$
i_{\beta}(\alpha ; d \theta)=e^{-\beta(\cosh \alpha-1)} e^{\beta[\cos (d \theta+i \alpha)-\cos (d \theta)]} .
$$

Using the identity

$$
\cos (\varphi+i \alpha)-\cos \varphi=\cos \varphi(\cosh \alpha-1)-i \sin \varphi \sinh \alpha,
$$

one sees that

$$
\max \left|i_{\beta}(\alpha ; \cdot)\right| \leqq 1
$$

Thus, the optimal choice of $\alpha$ in (3.32) apparently corresponds to minimizing

$$
-\alpha \varrho_{x y}+6 \beta(\cosh \alpha-1) \text {. }
$$


For our purposes it suffices to choose

$$
\alpha=\alpha_{x y} \equiv c_{0} \operatorname{sign} \varrho_{x y},
$$

hence

$$
e^{-\alpha \varrho_{x y}} e^{6 \beta(\cosh \alpha-1)} \leqq e^{c_{1} \beta-c_{0}\left|\varrho_{x y}\right|},
$$

where $c_{0}$ and $c_{1}$ are finite constants.

We now define

$$
\begin{aligned}
F(\varrho ; d \theta+\varepsilon)= & \frac{1}{2} e^{i\left(d \theta\left(\mu_{Q}\right)+\varepsilon\left(\mu_{\varrho}\right)\right)} \prod_{x y \subset \mathscr{B}_{\varrho}} \prod_{p: \partial p \supset x y} i_{\beta}\left(\alpha_{x y} ; d \theta_{p}+\varepsilon_{p}\right) \\
& +\frac{1}{2} e^{-i\left(d \theta\left(\mu_{\varrho}\right)+\varepsilon\left(\mu_{\varrho}\right)\right)} \prod_{x y \subset \mathscr{B}_{\varrho}} \prod_{p: \partial p \supset x y} i_{\beta}\left(-\alpha_{x y} ; d \theta_{p}+\varepsilon_{p}\right) .
\end{aligned}
$$

By (3.33), $F(\varrho ; d \theta+\varepsilon)$ is a real-valued function of $\theta$ which, by (3.34), is bounded in modulus by 1 and, for $\varepsilon \equiv 0$, is even in $\theta$. Furthermore, we define

$$
\begin{aligned}
z(\beta, \varrho) & \equiv K(\varrho) \prod_{x y \subset \mathscr{B}_{\varrho}} e^{c_{1} \beta-c_{0}\left|\varrho_{x y}\right|} \\
& \leqq K(\varrho) \exp (1 / 18)\left[c_{1} \beta L(\varrho)-c_{0}\|\varrho\|_{1}\right] .
\end{aligned}
$$

By repeating the arguments used in the proof of Corollary 4, Sect. 2.8, and making use of (3.31)-(3.33), (3.36), and (3.37) we obtain

$$
\mathscr{I}_{\gamma}(\varepsilon)=\int \prod_{\substack{\varrho \in \mathscr{N}_{\gamma}^{1} \\ \delta \varrho=0}}[1+z(\beta, \varrho) F(\varrho ; d \theta+\varepsilon)] \mathscr{R}(d \theta+\varepsilon) d \mu_{\beta}(\theta) .
$$

\subsection{The Lower Bound for $\mathscr{I}_{\gamma}(\varepsilon)$}

We now estimate $z(\beta, \varrho)$ and then prove a lower bound on $\mathscr{I}_{\gamma}(\varepsilon)$ which will establish our main result, the perimeter decay of the expectation value (3.28) of the disorder operator, $D_{\mathscr{L}_{L \times T}}^{\xi}$, for $N>N(\beta)$ and all finite values of $\beta$.

From the upper bound (3.37) on $z(\beta, \varrho)$ we derive, using inequalities (3.14) [bound on $z_{q N}$ ] and (3.19) [bound on $K(\varrho)$ ],

$$
\begin{aligned}
z(\beta, \varrho) & \leqq K(\varrho) \exp (1 / 18)\left[c_{1} \beta L(\varrho)-c_{0}\|\varrho\|_{1}\right] \\
& \leqq \exp \left[c_{2}(\beta) L(\varrho)-\left(c_{3} N-\varepsilon\right)(1 / N)\|\varrho\|_{1}\right]
\end{aligned}
$$

for some function $c_{2}(\beta) \leqq c_{1} \beta+c_{4}$ and some finite constants $c_{3}>0$ and $c_{4}$. It follows from the fact that a current distribution takes values in $N \mathbb{Z}$ that

$$
(1 / N)\|\varrho\|_{1} \geqq L(\varrho),
$$

so that if $N>1+\varepsilon / c_{3}$

$$
z(\beta, \varrho) \leqq \exp \left[\left(c_{2}(\beta)-c_{5} N\right) L(\varrho)-c_{6}\|\varrho\|_{1}\right]
$$

for some positive constants $c_{5}$ and $c_{6}$. [Given $\beta$ and $N$, one may now optimize in the choice of $\varepsilon$, see (3.12).]

Thus if $N>c_{1} \beta+c_{7}$, for some constant $c_{7}<\infty$,

$$
z(\beta, \varrho)<1
$$


and

$$
z(\beta, \varrho) \rightarrow 0, \quad \text { as } \quad N \rightarrow \infty,
$$

exponentially fast, for arbitrary $\beta<\infty$.

We now analyze the dependence on $\varepsilon_{p}$ of the integrand on the right side of expression (3.38) for $\mathscr{I}_{\gamma}(\varepsilon)$. For this purpose we rewrite the factors $1+z(\beta, \varrho) F(\varrho ; d \theta+\varepsilon)$, namely

$$
\begin{aligned}
1+z(\beta, \varrho) F(\varrho ; d \theta+\varepsilon)= & {[1+z(\beta, \varrho) F(\varrho ; d \theta)] } \\
& \cdot \exp \ln \left(1+\frac{z(\beta, \varrho)\{F(\varrho ; d \theta+\varepsilon)-F(\varrho ; d \theta)\}}{1+z(\beta, \varrho) F(\varrho ; d \theta)}\right),
\end{aligned}
$$

and apply Taylor's theorem with remainder to the functions $\ln (1+x)$ and $F(\varrho, d \theta+\varepsilon)-F(\varrho, d \theta)$. This yields

$$
\begin{aligned}
1+z(\beta, \varrho) F(\varrho ; d \theta+\varepsilon)= & {[1+z(\beta, \varrho) F(\varrho ; d \theta)] } \\
& \cdot \exp O_{\varrho}(\varepsilon ; d \theta) \exp R_{\varrho}(\varepsilon ; d \theta),
\end{aligned}
$$

where

$$
\left.O_{\varrho}(\varepsilon ; d \theta) \equiv \frac{\partial}{\partial \lambda} F(\varrho ; d \theta+\lambda \varepsilon)\right|_{\lambda=0} \cdot \frac{z(\beta, \varrho)}{1+z(\beta, \varrho) F(\varrho ; d \theta)},
$$

which is an odd function of $\theta$, because $F(\varrho ; d \theta)$ is even in $\theta$, and

$$
\begin{aligned}
R_{\varrho}(\varepsilon ; d \theta)= & -\frac{1}{2}\left(\frac{t \cdot z(\beta, \varrho)\{F(\varrho ; d \theta+\varepsilon)-F(\varrho ; d \theta)\}}{1+z(\beta, \varrho) F(\varrho ; d \theta)}\right)^{2} \\
& +\frac{1}{2} \frac{\left.z(\beta, \varrho) \frac{\partial^{2}}{\partial \lambda^{2}} F(\varrho ; d \theta+\lambda \varepsilon)\right|_{\lambda=s}}{1+z(\beta, \varrho) F(\varrho ; d \theta)},
\end{aligned}
$$

for some numbers $t$ and $s$ in the interval $(0,1)$. By inspecting the explicit expression (3.36) for $F(\varrho ; d \theta+\lambda \varepsilon)$ and estimating the first and second derivative in $\lambda$ one shows quite easily that

where

$$
\left|R_{\varrho}(\varepsilon ; d \theta)\right| \leqq k_{\varrho}(\varepsilon)^{2} z(\beta, \varrho)
$$

$$
k_{\varrho}(\varepsilon) \equiv C\left\{\left|\varepsilon\left(\mu_{\varrho}\right)\right|+\beta L(\varrho) \max _{p \in \operatorname{supp} \varrho}\left|\varepsilon_{p}\right|\right\},
$$

for some finite constant $C$, provided $N$ is chosen so large that $z(\beta, \varrho)<1 / 2$, for all $\varrho \in \mathscr{N}_{\gamma}^{1}$ and all $\gamma$. [By (3.40) this is the case for all sufficiently large $N$.]

Furthermore, from definition (3.30) of $\mathscr{R}(d \theta+\varepsilon)$, Sect. 3.3, and Taylor's theorem with remainder we derive

$$
\mathscr{R}(d \theta+\varepsilon)=e^{O(\varepsilon ; d \theta)} e^{R(\varepsilon ; d \theta)}
$$

where $O(\varepsilon ; d \theta)$ is an odd function of $\theta$, and

with

$$
\left.\begin{array}{l}
R(\varepsilon ; d \theta)=\sum_{p} R\left(\varepsilon_{p} ; d \theta_{p}\right) \\
\left|R\left(\varepsilon_{p} ; d \theta_{p}\right)\right| \leqq(\beta / 2) \varepsilon_{p}^{2}
\end{array}\right\}
$$


We now insert the right sides of (3.42) and (3.46) into (3.38) and subsequently apply estimates (3.45) and (3.47). This yields the following lower bound on $\mathscr{I}_{\gamma}(\varepsilon)$

$$
\begin{aligned}
& \mathscr{I}_{\gamma}(\varepsilon) \geqq e^{-(\beta / 2)\|\varepsilon\|_{2}^{2}} \prod_{\substack{\varrho \in \mathcal{N}^{1} \\
\delta \varrho=0}}\left[\exp -k_{\varrho}(\varepsilon)^{2} z(\beta, \varrho)\right] \\
& \cdot \int \prod_{\substack{\varrho \in \mathcal{N}^{1} \\
\delta \varrho=0}}[1+z(\beta, \varrho) F(\varrho ; d \theta)] e^{-o_{\varrho}(\varepsilon ; d \theta)} e^{-O(\varepsilon ; d \theta)} d \mu_{\beta}(\theta) .
\end{aligned}
$$

Since $\prod_{\substack{\varrho \in \mathcal{N}^{1} \\ \delta \varrho}}[1+z(\beta, \varrho) F(\varrho ; d \theta)] d \mu_{\beta}(\theta)$ is an even, positive measure in $\theta$ if $N$ is so large that $z(\beta, \varrho)<1$, for all $\varrho \in \mathcal{N}_{\gamma}^{1}$ and all $\gamma$, while $\sum_{\substack{\varrho \in \mathcal{N}^{1} \\ \delta \varrho}} O_{\varrho}(\varepsilon ; d \theta)$ and $O(\varepsilon ; d \theta)$ are odd functions of $\theta$, Jensen's inequality finally yields

$$
\sum_{\substack{\varrho \in \mathcal{N}^{1} \\ \delta \varrho}} O_{\varrho}(\varepsilon ; d \theta) \text { and } O(\varepsilon ; d \theta) \text { are }
$$

$$
\mathscr{I}_{\gamma}(\varepsilon) \geqq e^{-(\beta / 2)\|\varepsilon\|_{2}^{2}}\left\{\prod_{\substack{\varrho \in \mathscr{N}^{1} \\ \delta \varrho=0}} \exp \left[-k_{\varrho}(\varepsilon)^{2} z(\beta, \varrho)\right]\right\} \cdot \mathscr{I}_{\gamma}(0) .
$$

We now estimate $k_{\varrho}(\varepsilon)$.

Using Lemma 1, Sect. 2.3, we obtain

$$
\begin{aligned}
k_{\varrho}(\varepsilon) & \equiv C\left\{\left|\varepsilon\left(\mu_{\varrho}\right)\right|+\beta L(\varrho) \max _{p \in \text { supp } \varrho}\left|\varepsilon_{p}\right|\right\} \\
& \leqq C\left(\text { const }\|\varrho\|_{1} L(\varrho)^{4}+\beta L(\varrho)\right) \max _{p \in \Omega_{\varrho}}\left|\varepsilon_{p}\right| \\
& \leqq C_{1} \beta\|\varrho\|_{1} L(\varrho)^{4} \cdot \max _{p \in \Omega_{\varrho}}\left|\varepsilon_{p}\right|,
\end{aligned}
$$

see (2.104), Sect. 2.12, so that by repeating the arguments leading to (2.105) and (2.106) and inserting the upper bound (3.40) on $z(\beta, \varrho)$ we find

$$
\begin{aligned}
\sum_{\substack{\varrho \in \mathcal{N}_{\nu}^{1} \\
\delta \varrho=0}} k_{\varrho}(\varepsilon)^{2} z(\beta, \varrho) & \leqq C_{2} \beta \max _{\varrho \in \mathcal{N}_{\nu}^{1}}\left(\|\varrho\|_{1}^{2} e^{-c_{6}\|\varrho\|_{1}}\right) \sum_{L=4}^{\infty}\left\{L^{11} e^{\left(c_{2}(\beta)-c_{5} N\right) L}\right\}\|\varepsilon\|_{2}^{2} \\
& \leqq c(\beta, N)\|\varepsilon\|_{2}^{2},
\end{aligned}
$$

for some function $c(\beta, N)$ which tends to 0 , as $N \rightarrow \infty$, exponentially fast, for each $\beta<\infty$.

We now return to our basic identity (3.28) for the expectation value of the disorder operator and insert the lower bounds (3.49) and (3.50). This yields

$$
\begin{aligned}
\left\langle D_{\mathscr{L}_{L \times T}}^{\xi_{L}}\right\rangle_{\Lambda}(\beta, h) & =\sum_{\gamma} \lambda_{\gamma} \mathscr{I}_{\gamma}(\varepsilon) / \mathscr{I}_{\gamma}(0) \\
& \geqq \exp \left[-\{(\beta / 2)+c(\beta, N)\}\|\varepsilon\|_{2}^{2}\right],
\end{aligned}
$$

where

$$
\lambda_{\gamma} \equiv\left(Z_{\beta, \Lambda}^{h}\right)^{-1} d_{\gamma} \mathscr{I}_{\gamma}(0), \text { hence } \sum \lambda_{\gamma}=1 \text {, }
$$

and

$$
\mathscr{I}_{\gamma}(\varepsilon)=\int \prod_{\substack{\varrho \in \mathscr{N}(1) \\ \delta \varrho=0}}[1+z(\beta, \varrho) F(\varrho ; d \theta+\varepsilon)] \mathscr{R}(d \theta+\varepsilon) d \mu_{\beta}(\theta)
$$


By (3.22) and (3.25), (3.26)

$$
\|\varepsilon\|_{2}^{2} \geqq \operatorname{const}(\xi / N)^{2}(L+T) .
$$

Thus, for arbitrary $h \leqq \infty$ and $\Lambda \subset \mathbb{Z}^{4}$,

$$
\left\langle D_{\mathscr{L}_{L \times T}}^{\xi}\right\rangle_{\Lambda}(\beta, h) \geqq e^{-\operatorname{const} \beta(\xi / N)^{2}(L+T)},
$$

for each $\beta<\infty$ and $N>N(\beta)$, for some function $N(\beta)<\infty$ (with $N(\beta) \uparrow \infty$, as $\beta \uparrow \infty$ ). This completes our proof of the lower bounds in (3.1).

Remarks. 1) The main results of this section are identities (3.51) and (3.52), the bounds (3.49) and (3.50) and the final inequality (3.54).

2) Identities (3.51) and (3.52) relate $\left\langle D_{\mathscr{L}_{L \times T}}\right\rangle_{A}(\beta, h)$ to (a convex combination of) expectation values of an observable, somewhat analogous to the disorder operator, in the measures

$$
\mathscr{I}_{\gamma}(0)^{-1} \prod_{\substack{\rho \in \mathcal{S}^{\prime} \\ \delta \varrho=0}}[1+z(\beta, \varrho) F(\varrho ; d \theta)] d \mu_{\beta}(\theta)
$$

which correspond to lattice gauge theories invariant under U(1) gauge transformations. [The observable is defined as the substitution

$$
d \theta \mapsto d \theta+\varepsilon,
$$

to be compared with definition (1.14) of disorder operators. It can be viewed as a renormalized disorder operator.]

The same comments apply to $\left\langle W\left(\mathscr{L}_{L \times T}\right)\right\rangle_{A}(\beta, h)$, but we do not wish to present the appropriate renormalization transformations for this expectation in the present paper. (See however [15] for the solution of a similar problem concerning correlations of fractional charges in a two-dimensional Coulomb gas.)

3) The techniques presented in this section can be extended to $\mathbb{Z}_{N}$ gauge theories in dimension $\geqq 3$.

\section{Transitions in Classical $X Y$ Models and "Hypergauge Theories"}

\subsection{Definition of Models}

In this section we comment on the phase diagram of a general class of $U(1)$ lattice models and their duals which are natural generalizations of the $X Y$ model and the $U(1)$ lattice gauge theory. They are of some interest for the statistical mechanics of defect gases. For the group $\mathbb{Z}_{2}$ such a class of models (generalizations of the Ising model and the $\mathbb{Z}_{2}$ lattice gauge theory) were first studied by Wegner in his basic paper [25].

As a byproduct we obtain results on the phase transition in three- or higher dimensional classical $X Y$ models, and, by combining the results of this section and of [15] with correlation inequalities [17, 24], some of the essential features of the phase diagram of abelian Higgs lattice theories in three and four dimensions can be established; see [5].

Thus, for the classical $X Y$ model [11] and the Villain approximation in three or more dimensions we find a proof of existence of a phase transition, accom- 
panied by spontaneous breaking of $U(1)$ and the appearance of a Goldstone excitation, and for the Higgs models we conclude the existence of a superconductor $\rightarrow$ QED transition, [5].

A rank- $k U(1)$ lattice theory is defined as follows: The configurations of a rank- $k U(1)$ lattice theory are functions

$$
\theta: c_{k} \mapsto \theta\left(c_{k}\right) \in S^{1}
$$

defined on $k$-cells, $c_{k}$, in $\mathbb{Z}^{D}$ with values in the unit circle, identified with $[-\pi, \pi)$, and with the property that

$$
\theta\left(c_{k}\right)=-\theta\left(c_{k}^{-}\right),
$$

where $c_{k}^{-}$is the same $k$-cell as $c_{k}$, but with reversed orientation; see Sect. 2.3.

We set

$$
d \theta\left(c_{k+1}\right)=\sum_{c_{k} \subset \partial c_{k+1}} \theta\left(c_{k}\right),
$$

where the orientation of $c_{k}$ is the one prescribed by the orientation of $c_{k+1}$.

Let $\varphi_{\beta}$ be a function on $S^{1}$ of positive type, e.g.

$$
\varphi_{\beta}(\theta)=\left\{\begin{array}{l}
\exp \beta \cos \theta, \\
\sum_{\ell=-\infty}^{\infty} \exp \left[-\frac{\beta}{2}(\theta+2 \pi \ell)^{2}\right] .
\end{array}\right.
$$

The vacuum functional (equilibrium state) of a rank- $k U(1)$ lattice theory with inverse square coupling (inverse temperature) $\beta$ in a finite region $\Lambda \subset \mathbb{Z}^{D}$ is given by

$$
d \mu_{\beta}\left(\theta_{\Lambda}\right) \equiv \tilde{Z}_{\beta, \Lambda}^{-1} \prod_{c_{k+1} \subset A} \varphi_{\beta}\left(d \theta\left(c_{k+1}\right)\right) \prod_{c_{k} \subset A} d \theta\left(c_{k}\right),
$$

where $\tilde{Z}_{\beta, \Lambda}$ is the usual partition function.

We propose to derive the phase diagram and the lower critical dimension, $D_{c}$, of rank- $k U(1)$ lattice theories. We claim that

$$
D_{c}=k+3
$$

except for $k=0(X Y$ model $)$ where

$$
D_{c}=2 ; \text { see }[15] \text {. }
$$

A natural observable to analyze is the following: Let $S_{k}$ be some closed, oriented surface built out of $k$-cells in $\mathbb{Z}^{D}$. We define

$$
W_{m}\left(S_{k}\right)=\prod_{c_{k} \subset S_{k}} e^{\operatorname{im} \theta\left(c_{k}\right)}
$$

and

$$
\left\langle W_{m}\left(S_{k}\right)\right\rangle_{\Lambda}(\beta) \equiv \int d \mu_{\beta}\left(\theta_{\Lambda}\right) W_{m}\left(S_{k}\right) .
$$

$W_{m}$ is the analogue of the Wegner-Wilson loop. Let $\Sigma_{k+1}$ be a bounded, $(k+1)$ dimensional region in $\mathbb{Z}^{D}$ built out of oriented $(k+1)$-cells with boundary $\partial \Sigma_{k+1}=S_{k}$. By (4.2) and (4.7)

$$
W_{m}\left(S_{k}\right)=\prod_{c_{k}+1 \subset \Sigma_{k+1}} e^{\mathrm{im} d \theta\left(c_{k+1}\right)} .
$$


Note that, for $k>1, d \mu_{\beta}\left(\theta_{\Lambda}\right)$ and $W_{m}\left(S_{k}\right)$ are invariant under the gauge transformations

$$
\begin{gathered}
\theta\left(c_{k}\right) \mapsto \theta\left(c_{k}\right)+d \omega\left(c_{k}\right), \\
d \omega\left(c_{k}\right)=\sum_{c_{k-1} \subset \partial c_{k}} \omega\left(c_{k-1}\right),
\end{gathered}
$$

where $\omega$ is an arbitrary function defined on the $(k-1)$-cells in $\mathbb{Z}^{D}$ with values in $S^{1}$.

When $k=0$, i.e. for the classical $X Y$ model, $S_{k=0}=\{x, y\}$, (two sites in $\mathbb{Z}^{D}$ ),

$$
W_{m}\left(S_{0}\right)=e^{\mathrm{im}\left(\theta_{x}-\theta_{y}\right)}=\prod_{b \in \Sigma_{1}} e^{\mathrm{im} d \theta_{b}},
$$

where $\Sigma_{1}$ is a line of oriented links joining $x$ to $y$, and gauge invariance is replaced by invariance under the global symmetry

$$
\theta_{x} \rightarrow \theta_{x}+\omega, \quad \omega \in[-\pi, \pi) .
$$

For the $X Y$ model, $D_{c}=2$ (see [15]).

The results of this section concern the models in $D \geqq 3$ dimensions which have the property that the dual models are $\mathbb{Z}$ (hyper)gauge theories to which our methods apply.

The methods of Sect. 3 permit us to also study rank- $k \mathbb{Z}_{N}$-models in dimension $D \geqq k+3$. (They are defined in the obvious way.) As in Sect. 3 one can prove the existence of intermediate phases, for sufficiently large $N$.

\subsection{The Duality Transformation}

Our analysis of rank- $k U(1)$ theories relies on a duality transformation. Let $\hat{\varphi}_{c_{k+1}}(n), n \in \mathbb{Z}$, denote the $n^{\text {th }}$ Fourier coefficient of a function $\varphi_{c_{k+1}}(\theta)$ on $S^{1}$. By Fourier transformation

$$
\begin{array}{r}
\int \prod_{c_{k+1} \subset A} \varphi_{c_{k+1}}\left(d \theta\left(c_{k+1}\right)\right) \prod_{c_{k} \subset A} d \theta\left(c_{k}\right) \\
=\sum_{n: \delta n=0} \prod_{c_{k+1} \subset A} \hat{\varphi}_{c_{k+1}}\left(n\left(c_{k+1}\right)\right),
\end{array}
$$

where each $n$ is a divergence-free, integer-valued $(k+1)$-form with support in $\Lambda$ [see (2.19)]. Given some integer-valued $(k+1)$-form $n$, $\operatorname{supp} n \subset \Lambda$, there exists an integer-valued $(k+2)$-form, $\mathrm{m}$, with

$$
n=\delta m, \text { and } \operatorname{supp} m \subset \Lambda
$$

[see Lemma 1, Sect. 2.3]. ( $\Lambda$ is assumed to have trivial homology. The multiplicity of solutions, $m$, of (4.14) is then independent of $n$. For details concerning the special case $D=2, k=1$ see Appendix A of [15].) We define

$$
\alpha=* m,
$$

which is a $k^{*} \equiv D-k-2$ form. Thus

$$
\begin{array}{r}
\int \prod_{c_{k+1} \subset A} \varphi_{c_{k+1}}\left(d \theta\left(c_{k+1}\right)\right) \prod_{c_{k} \subset A} d \theta\left(c_{k}\right) \\
=\sum_{[\alpha]} \prod_{c_{k+1} \subset \Lambda} \hat{\varphi}_{c_{k+1}}\left((* d \alpha)\left(c_{k+1}\right)\right),
\end{array}
$$


where $\sum_{[\alpha]}$ ranges over all equivalence classes of integer-valued $k^{*}$-forms, $\alpha$, with

$$
n=* d \alpha, \quad \operatorname{supp} \alpha \subset \Lambda^{*}
$$

Applications. 1) $\varphi_{c_{k+1}}=\varphi_{\beta}$, for all $c_{k+1} \subset \Lambda$. This yields

$$
\tilde{Z}_{\beta, \Lambda}=\sum_{[\alpha]} \prod_{c_{k+1} \subset A} \hat{\varphi}_{\beta}\left((* d \alpha)\left(c_{k+1}\right)\right) .
$$

2)

$$
\varphi_{c_{k+1}}= \begin{cases}\varphi_{\beta}, & c_{k+1} \subset \Lambda \sim \Sigma_{k+1} \\ e^{\operatorname{im} \theta} \varphi_{\beta}, & c_{k+1} \subset \Sigma_{k} .\end{cases}
$$

With (4.18) this yields

$$
\begin{aligned}
& \left\langle W_{m}\left(S_{k}\right)\right\rangle_{\Lambda}(\beta) \\
& \quad=\tilde{Z}_{\beta, \Lambda}^{-1}\left\{\sum_{[\alpha]} \prod_{c_{k+1} \subset \Lambda \sim \Sigma_{k+1}} \hat{\varphi}_{\beta}\left((* d \alpha)\left(c_{k+1}\right)\right) \prod_{c_{k+1} \subset \Sigma_{k+1}} \hat{\varphi}_{\beta}\left((* d \alpha)\left(c_{k+1}\right)-m\right)\right\} .
\end{aligned}
$$

As an example, we consider the rank- $k$ Villain models. One chooses

$$
\varphi_{\beta}(\theta)=\sum_{\ell=-\infty}^{\infty} \exp \left[-\frac{\beta}{2}(\theta+2 \pi \ell)^{2}\right]
$$

i.e.

$$
\hat{\varphi}_{\beta}(n)=\text { const } \exp \left[-n^{2} / 2 \beta\right] \text {. }
$$

Then

$$
\left\langle W_{m}\left(S_{k}\right)\right\rangle_{\Lambda}(\beta)=\hat{Z}_{\beta, \Lambda}^{-1}\left\{\sum_{[\alpha]} \prod_{\left(c_{k^{*}}\right)^{*} \subset \Lambda^{*}} \exp \left[-\frac{1}{2}(d \alpha+\varphi)\left(c_{k^{*}+1}^{*}\right)^{2}\right]\right\},
$$

where

$$
\varphi\left(c_{k^{*}+1}^{*}\right)= \begin{cases}-m, & c_{k+1} \equiv\left(c_{k^{*}+1}^{*}\right)^{*} \subset \Sigma_{k+1} \\ 0, & \text { otherwise }\end{cases}
$$

For the three-dimensional Villain model $(k=0)$ we obtain

$$
\left\langle W_{1}\left(S_{0}\right)\right\rangle_{\Lambda}(\beta)=\left\langle e^{i\left(\theta_{x}-\theta_{y}\right)}\right\rangle_{\Lambda}(\beta)=\left\langle\mathbf{S}_{x} \cdot \mathbf{S}_{y}\right\rangle_{\Lambda}(\beta),
$$

so that

$$
\left\langle\mathbf{S}_{x} \cdot \mathbf{S}_{y}\right\rangle_{\Lambda}(\beta)=\hat{Z}_{\beta, \Lambda}^{-1}\left\{\sum_{[\alpha]} \prod_{p \subset \Lambda^{*}} \exp \left[-\frac{1}{2 \beta}(d \alpha+\varphi)(p)^{2}\right]\right\},
$$

where

$$
\varphi(p)= \begin{cases}-1, & \text { if } b \equiv p^{*} \subset \Sigma_{1} \\ 0, & \text { otherwise }\end{cases}
$$

and $\Sigma_{1}$ is a path of links, $b$, (dual to plaquettes for $D=3$ ) joining $x$ to $y$. 
Remark. One may also introduce disorder operators, $D_{\partial S_{D-k-1}}^{\xi}$, for rank-k $U(1)$ theories, in analogy with (1.14) and (3.21). It is easy to show that

$$
\left\langle D_{\partial S_{D-k-1}}^{\xi}\right\rangle_{\Lambda}(\beta)=\tilde{Z}_{\beta, \Lambda}^{-1}\left\{\sum_{[\alpha]} \prod_{c_{k+1} \subset \Lambda} \hat{\varphi}_{\beta}\left((* d \alpha)\left(c_{k+1}\right)\right) \prod_{c_{k}^{* *}\left(\partial\left(\partial S_{D-k-1}\right)^{*}\right.} e^{i \xi \alpha\left(c_{k^{*}}^{*}\right)}\right\} .
$$

For $k>0$ and $\varphi_{\beta}$ as in (4.20)

$$
\left\langle D_{\partial S_{D-k-1}}^{\zeta}\right\rangle_{\Lambda}(\beta) \geqq \exp \left[-\operatorname{const} \operatorname{vol}\left(\partial S_{D-k-1}\right)\right] .
$$

This follows from the result for the Gaussian expectation value, by using the correlation inequalities of [24]. For the Villain model $(k=0),\left\langle D_{\partial S_{D-1}}^{\xi}\right\rangle_{\Lambda}(\beta)$ is related to the surface tension which vanishes in the thermodynamic limit. The asymptotic behaviour for large $S_{D-1}, \Lambda \uparrow \mathbb{Z}^{D}$, can be determined by combining the results of [15] $(k=0, D=2)$ with correlation inequalities. See [5] for the threedimensional model.

\subsection{The Main Results}

We now study the expectation value $\left\langle W_{m}\left(S_{k}\right)\right\rangle_{\Lambda}(\beta)$ for a rank- $k$ Villain model. As in Sect. 2.6 we reexpress the dual model in terms of a Gaussian measure, $d \mu_{\Lambda}^{0}(\alpha)$, defined, for $D-k-2 \geqq 1$, i.e. $D \geqq k+3$, by

$$
\int d \mu_{\Lambda}^{0}(\alpha) e^{i \alpha(\mu)}= \begin{cases}\exp \left[-\frac{\beta}{2}\left(\mu, V_{\Lambda} \mu\right)\right], & \text { if } \delta \mu=0 \\ 0, & \text { otherwise }\end{cases}
$$

where $V_{A}$ is the Green's function of $\Pi_{\Lambda^{*}} \delta d$ [see (2.34), Sect. 2.5]. When $D-k-2=0, \alpha$ is a scalar lattice field, and $d \mu_{\Lambda}^{0}(\alpha)$ is the usual Gaussian measure with Dirichlet b.c. at $\partial \Lambda$. In this case, the dual of the rank- $k$ Villain model is isomorphic to a $D$-dimensional Coulomb gas. For $D=2$ this gas is analyzed in [15], where it is shown that it exhibits a Kosterlitz-Thouless transition. For $D \geqq 3$, it is believed that there are no bulk phase transitions in this gas and that it exhibits Debye screening [10], for all values of $\beta$. [This is because the Coulomb potential behaves like dist ${ }^{-D+2}$, for $D \geqq 3$, while in $D=2$ it behaves like $\log$ (dist).] The main result of this section is that when

$$
D>k+2
$$

the rank- $k$ Villain model has a massive small $\beta$ phase in which

$$
\left\langle W_{m}\left(S_{k}\right)\right\rangle(\beta) \leqq \exp \left[- \text { const } \operatorname{vol}\left(\Sigma_{k+1}^{0}\right)\right],
$$

where $\Sigma_{k+1}^{0}$ is a minimal region with $\partial \Sigma_{k+1}^{0}=S_{k}$; (this follows from a standard high temperature expansion), and a massless large $\beta$ phase where

$$
\left\langle W_{m}\left(S_{k}\right)\right\rangle(\beta) \geqq \exp \left[- \text { const } \operatorname{vol}\left(S_{k}\right)\right] .
$$

The proof of (4.27) is a straightforward variant of the one in Sects. 2.4-2.10 which we sketch below.

In conclusion, the lower critical dimension is

$$
D_{c}=k+3, \text { for } k \geqq 1 \text {. }
$$




\subsection{Sketch of the Proof}

As in Sect. 2.6 one shows that, for a rank- $k$ Villain model,

$$
\begin{aligned}
& \left\langle W\left(S_{k}\right)\right\rangle_{\Lambda}(\beta) \\
& \quad=Z_{\beta, \Lambda}^{-1} \int \prod_{c^{*} \subset \Lambda^{*}}\left\{1+2 \sum_{(2 \pi)^{-1} q=1}^{\infty} \cos \left(q \alpha\left(c_{k^{*}}\right)\right)\right\} \mathscr{R}_{\varphi}(d \alpha) d \mu_{\Lambda}^{0}(\alpha),
\end{aligned}
$$

where

$$
\mathscr{R}_{\varphi}(d \alpha)=\prod_{c_{k^{*} C} \Lambda^{*}} \exp \left[-\frac{1}{2 \beta}\{2(d \alpha, \varphi)+(\varphi, \varphi)\}\right]
$$

We now apply the combinatorial expansion of Sect. 2.6 to

$$
I\left(\alpha_{\Lambda}\right) \equiv \prod_{c_{k *} \subset \Lambda^{*}}\left\{1+2 \sum_{(2 \pi)^{-1} q=1}^{\infty} \cos \left(q \alpha\left(c_{k^{*}}\right)\right)\right\} .
$$

We define a rank- $k^{*}$ current distribution, $\varrho$, as a function on $\left(k^{*} \equiv D-k-2\right)$-cells in $\Lambda^{*}$ with values in $2 \pi \mathbb{Z}$.

By mimicking the combinatorial scheme of Sect. 2.6 we obtain

$$
I\left(\alpha_{\Lambda}\right)=\sum_{\gamma} d_{\gamma} \prod_{\varrho \in \mathcal{N}_{\gamma}^{1}}[1+K(\varrho) \cos \alpha(\varrho)]
$$

where $\gamma$ ranges over some finite index set, each $\mathscr{N}_{\gamma}^{1}$ is a 1 -ensemble, [i.e. $\operatorname{dist}\left(\varrho_{1}, \varrho_{2}\right) \geqq \sqrt{2}$, for two distinct rank- $k^{*}$ current distributions $\varrho_{1}$ and $\varrho_{2}$ in $\left.\mathscr{N}_{\gamma}^{1}\right]$, and

i) $c_{\gamma}>0$, for all $\gamma$

ii) $0<K(\varrho) \leqq 3^{N_{1}(\operatorname{supp} \varrho)} \prod_{c_{k} \star C \operatorname{supp} \varrho} z_{\left|\varrho\left(c_{k}\right)\right|}$,

where $N_{1}(\operatorname{supp} \varrho)$ is the number of $k^{*}$-cells within distance $\leqq 1$ of $\operatorname{supp} \varrho$, and

$$
z_{q}=e^{\beta_{0} q^{2}},
$$

for some constant $\beta_{0}$ with the property that

$$
\sum_{(2 \pi)^{-1} q=1}^{\infty} e^{-\beta_{0} q^{2}}=1 / 2 .
$$

Thus

$$
\left\langle W_{m}\left(S_{k}\right)\right\rangle_{\Lambda}(\beta)=Z_{\beta, \Lambda}^{-1}\left\{\sum_{\gamma} d_{\gamma} \int \prod_{\varrho \in \mathcal{N}^{1} \frac{1}{\gamma}}[1+K(\varrho) \cos \alpha(\varrho)] \mathscr{R}_{\varphi}(d \alpha) d \mu_{\Lambda}^{0}(\alpha)\right\} .
$$

Because of (4.25) we may omit all factors from the right side of (4.32) for which $\delta \varrho \neq 0$, provided $D \geqq k+3$. (See [15] for $D=2, k=0$.)

Next, we change variables:

where

$$
\left.\begin{array}{c}
\alpha \rightarrow \alpha+\tau \\
\tau=\delta \Delta_{\Lambda}^{-1} \varphi
\end{array}\right\}
$$


and $\varphi$ is given by (4.21). Since

with

$$
\left.\begin{array}{rl}
\Pi_{\Lambda^{*}} d \tau & =-\varphi+\varepsilon_{\Lambda} \\
\varepsilon_{\Lambda} & =-\Pi_{\Lambda^{*}} \delta d \Delta_{\Lambda}^{-1} \varphi
\end{array}\right\}
$$

we obtain, using Lemma 1, Sect. 2.3, and the periodicity of the cosine,

$$
\left\langle W_{m}\left(S_{k}\right)\right\rangle_{\Lambda}(\beta)=Z_{\beta, \Lambda}^{-1}\left\{\sum_{\gamma} d_{\gamma} \int \prod_{\substack{\varrho \in \mathcal{N}_{\gamma}^{1} \\ \delta \varrho=0}}\left[1+K(\varrho) \cos \left(\alpha(\varrho)+\varepsilon_{\Lambda}\left(\mu_{\varrho}\right)\right)\right] d \mu_{\Lambda}^{0}\left(\alpha+\varepsilon_{\Lambda}\right)\right\} .(4.3
$$

The renormalization of the right side of (4.35) is performed as in Sect. 2.8 (see also Sect. 3.5, and Sect. 4 of [15]). It yields

$$
\left\langle W_{m}\left(S_{k}\right)\right\rangle_{\Lambda}(\beta)=Z_{\beta . \Lambda}^{-1}\left\{\sum_{\gamma} d_{\gamma} \int \prod_{\substack{\varrho \in \mathcal{N}^{1} \\ \delta \varrho=0}}\left[1+z(\beta, \bar{\varrho}) \cos \left(\alpha(\bar{\varrho})+\varepsilon_{\Lambda}\left(\mu_{\varrho}\right)\right] d \mu_{\Lambda}^{0}\left(\alpha+\varepsilon_{\Lambda}\right)\right\}^{(4.36)}\right.
$$

where

$$
z(\beta, \bar{\varrho}) \leqq \exp \left[\left(c_{1}-d_{1} \beta\right)\|\varrho\|_{2}^{2}\right] \exp \left[\left(c_{2}-d_{2} \beta\right) L(\varrho)\right],
$$

for $\beta>\max \left(c_{1} / d_{1}, c_{2} / d_{2}\right)$. Here $L(\varrho)$ is the number of $\left(k^{*} \equiv D-k-2\right)$-cells in supp $\varrho$, and $c_{1}, c_{2}, d_{1}$, and $d_{2}$ are finite, positive constants. A straightforward variant of the estimates in Sect. 2.10 and of (2.104)-(2.107), Sect. 2.11, yields.

$$
\left\langle W_{m}\left(S_{k}\right)\right\rangle_{\Lambda}(\beta) \geqq \exp \left[-\frac{1}{2 \beta^{\prime}}\left(\varepsilon_{\Lambda}, \varepsilon_{\Lambda}\right)\right],
$$

provided $\beta$ is sufficiently large. Here

$$
\frac{1}{2 \beta^{\prime}} \equiv \frac{1}{2 \beta}+d(\beta)
$$

where $d(\beta)$ is a finite function which tends to 0 , as $\beta \rightarrow \infty$, exponentially fast [see (2.80) and (2.87), (2.88), Sect. 2.10]. Finally, from (4.34) and the fact that the gradient of the Green's function of the Laplacian, $\Delta=-(d \delta+\delta d)$, decays like, $(1 / \text { dist })^{D-1}$, we conclude that

$$
\lim _{\Lambda \uparrow \mathbb{Z}^{D}}\left(\varepsilon_{\Lambda}, \varepsilon_{\Lambda}\right) \leqq \operatorname{const} \operatorname{vol}\left(S_{k}\right)
$$

for $D \geqq 3$. This completes our sketch of the proof of (4.27).

In the example of the three (or higher) dimensional Villain model $(k=0)$ we obtain from (4.38), (4.39), and (4.23)

$$
\begin{aligned}
\left\langle\mathbf{S}_{x} \cdot \mathbf{S}_{y}\right\rangle(\beta) & =\lim _{\Lambda \uparrow \mathbb{Z}^{D}}\left\langle\mathbf{S}_{x} \cdot \mathbf{S}_{y}\right\rangle_{\Lambda}(\beta) \\
& \geqq \exp \left[-\frac{1}{2 \beta^{\prime}} \operatorname{const} \operatorname{vol}\left(S_{0}\right)\right] \\
& =\exp (-C / \beta),
\end{aligned}
$$

for some finite constant $C$ independent of $x$ and $y$, provided $D \geqq 3$ and $\beta$ is sufficiently large. (The limit $\Lambda \nearrow \mathbb{Z}^{D}$ exists, as follows from Ginibre's inequalities.) 
Inequality (4.40) expresses long range order in the spin-spin correlation of the Villain model, for sufficiently large values of $\beta$. Thus, in the pure phases obtained by ergodic decomposition of $\langle-\rangle(\beta)$, the continuous, global $U(1)$ symmetry is broken.

The masslessness of the large $\beta$ phases of rank- $k$ Villain models, with $D \geqq k+3$, can be proven by generalizing the techniques developed in Sects. 2.11 and 2.12 in a straightforward way.

The techniques of our paper do not depend upon imposing special b.c. (They apply to a very large class of $U(1)$-invariant b.c., see e.g. Appendix A of [15] for a discussion of such b.c. for the two-dimensional, classical $X Y$ model.) None of our estimates relies on translation invariance. Using the tools in Sects. 6 and 7 and Appendix B and C of [15], we can extend our results to a fairly large class of functions, $\varphi_{\beta}$, in particular

$$
\varphi_{\beta}(\theta)=\exp \beta \cos \theta .
$$

These are definite advantages over the methods of [11] which rely on translation invariance and reflection positivity. (Those methods do, however, permit one to analyze spin systems with non-abelian symmetry groups for which no useful notion of duality exists, such as the classical Heisenberg model.)

We believe that our methods ought to be useful for the analysis of the quantum mechanical $X Y$ model, models of interacting Bose gases and statistical mechanical models of defects and dislocations in ordered media.

\section{References}

1. Wilson, K.: Phys. Rev. D10, 2445 (1974)

Osterwalder, K., Seiler, E.: Ann. Phys. (NY) 110, 440 (1978)

2. Gallavotti, G., Guerra, F., Miracle-Solé, S.: In: Mathematical problems in theoretical physics. Dell'Antonio, G., Doplicher, S., Jona-Lasinio, G. (eds.). Lecture Notes in Physics, Vol. 80. Berlin, Heidelberg, New York: Springer 1978

3. Mack, G., Petkova, V.: Ann. Phys. (NY), 123, 442 (1979); 125, 117 (1980)

4. Fröhlich, J.: Phys. Lett. 83B, 195 (1979)

5. Brydges, D., Fröhlich, J., Seiler, E.: Nucl. Phys. B152, 521 (1979)

6. Seiler, E.: Gauge theories as a problem in constructive quantum field theory. In: Lecture Notes in Physics. Berlin, Heidelberg, New York: Springer 1982 (to appear)

7. Balian, R., Drouffe, J.-M., Itzykson, C.: Phys. Rev. D10, 3376 (1974); D11, 2098 (1975); D11, 2104 (1975)

8. Gallavotti, G., Martin-Löf, A., Miracle-Solé, S.: In: Statistical Mechanics and Mathematical Problems. Lenard, A. (ed.). Lecture Notes in Physics, Vol. 20. Berlin, Heidelberg, New York: Springer 1973

Malyshev, S.: Commun. Math. Phys. 64, 131 (1979)

9. Glimm, J., Jaffe, A., Spencer, T.: Ann. Phys. (N.Y.) 101, 610 (1976); 101, 631 (1976)

10. Brydges, D.: Commun. Math. Phys. 58, 313 (1978) Brydges, D., Federbush, P.: Commun. Math. Phys. 73, 197 (1980)

12. Hasenfratz, A., Hasenfratz, E., Hasenfratz, P.: Nucl. Phys. B180, 353 (1981)

Itzykson, C., Peskin, M.E., Zuber, J.-B. : Phys. Lett. 95 B, 259 (1980)

Münster, G., Weisz, P.: Nucl. Phys. B180, 330 (1981)

Fröhlich, J.: In: Common trends in particle and condensed matter physics, ed. by Brézin, E., Gervais, J.-L., Toulouse, G.: Phys. Rep. 67, 137 (1980)

13. Guth, A.: Phys. Rev. D21, 2291 (1980) 
14. Berezinskii, V.L. : Sov. Phys. JETP 32, 493 (1971)

Kosterlitz, J.M., Thouless, D.J.: J. Phys. C6, 1181 (1973)

Kosterlitz, J.M. : J. Phys. C7, 1046 (1974)

15. Fröhlich, J., Spencer, T.: Phys. Rev. Lett. 46, 1006 (1981); Commun. Math. Phys. 81, 527 (1981)

16. Elitzur, S., Pearson, R., Shigemitsu, J. : Phys. Rev. D19, 3698 (1979)

17. Ginibre, J.: Commun. Math. Phys. 16, 310 (1970); also: de Angelis, G.F., de Falco, D., Guerra, F., Marra, R.: Acta Phys. Aust. Suppl. XIX, 205 (1978)

18. See Osterwalder, K., Seiler, E. : [1], and Durhuus, B., Fröhlich, J.: Commun. Math. Phys. 75, 103 (1980)

19. Seiler, E.: Phys. Rev. D18, 482 (1978)

20. Glimm, J., Jaffe, A.: Commun. Math. Phys. 56, 195 (1977)

21. Fröhlich, J., Spencer, T.: J. Stat. Phys. 24, 617 (1981)

22. Simon, B.: Commun. Math. Phys. 77, 111 (1980)

23. Tomboulis, A., Ukawa, Windey, P.: Princeton preprint 1980

24. Fröhlich, J.: In [2], Brydges, D., Fröhlich, J., Seiler, E.: Ann. Phys. (N.Y.) 121, 227 (1979)

25. Wegner, F.: J. Math. Phys. 12, 2259 (1971)

Communicated by A. Jaffe

Received September 19, 1981 